\title{
Spin-polarized Quantum Transport in Mesoscopic Conductors: Computational Concepts and Physical Phenomena
}

\author{
Michael Wimmer, Matthias Scheid, and Klaus Richter \\ Institut für Theoretische Physik, Universität Regensburg, D-93040 Regensburg, Germany
}

\section{Contents}

I. Definition of the subject and its importance

II. Introduction

III. Numerical quantum transport

A. Landauer-Büttiker transport theory

B. Tight-binding representation of the Hamiltonian

C. Numerical algorithms

IV. Matrix reordering strategies for quantum transport

A. Graph-theoretical approaches to matrix reordering

B. Example: ring geometry

V. Spin filtering in nanostructures

VI. Pure spin current generation

VII. Future directions

VIII. Acknowledgements

References

Glossary

\section{Aharonov Bohm effect}

The magnetic flux enclosed in between propagating quantum mechanical waves shifts their relative phases as a result of the underlying electromagnetic vector potential. This gives rise to distinct oscillations in the magnetoconductance of a ring conductor.

\section{Landauer-Büttiker formalism}

For phase-coherent quantum transport, the Landauer-Büttiker formalism relates the conductance of a device to the transmission probability of charge carriers.

Rashba- and Dresselhaus spin-orbit coupling

Coupling of the spin degree of freedom to the orbital motion of charge carriers due to structural or bulk inversion asymmetry in semiconductors.

\section{Ratchets}

Devices that convert unbiased fluctuations or perturbations into directed motion.

\section{Spintronics}

Extension of charge-based electronics in metals or semiconductors by utilizing the spin degree of freedom of the charge carriers. 


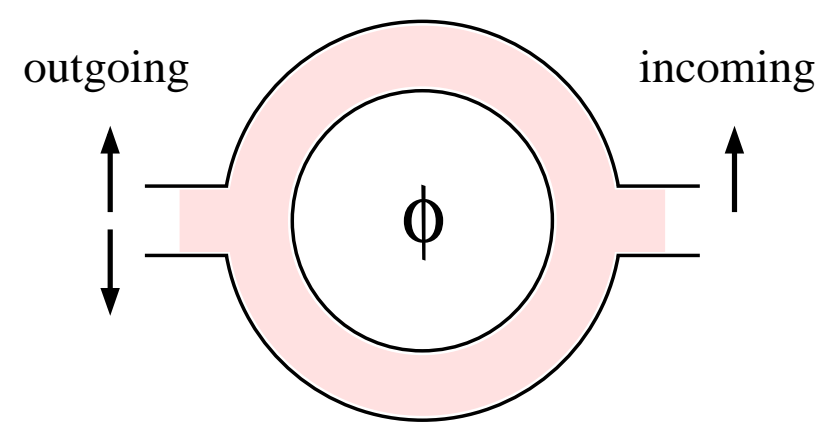

FIG. 1: Aharonov-Bohm physics with spin: Two-dimensional Aharonov-Bohm ring of mean radius $r_{0}$ used for numerical calculations of the conductance presented in Fig. 2. An additional perpendicular magnetic field $\mathbf{B}$ generates a flux $\phi=\pi r_{0}^{2} B$. The grey zone corresponds to the region subject to a finite Rashba coupling switched adiabatically on and off in the leads (from Ref. [4]).

\section{DEFINITION OF THE SUBJECT AND ITS IMPORTANCE}

Mesoscopic conductors are electronic systems of sizes in between nano- and micrometers, and often of reduced dimensionality. In the phase-coherent regime at low temperatures, the conductance of these devices is governed by quantum interference effects, such as the Aharonov-Bohm effect and conductance fluctuations as prominent examples. While first measurements of quantum charge transport date back to the 1980s, spin phenomena in mesoscopic transport have moved only recently into the focus of attention, as one branch of the field of spintronics. The interplay between quantum coherence with confinement-, disorder- or interaction-effects gives rise to a variety of unexpected spin phenomena in mesoscopic conductors and allows moreover to control and engineer the spin of the charge carriers: spin interference is often the basis for spin-valves, -filters, -switches or -pumps. Their underlying mechanisms may gain relevance on the way to possible future semiconductor-based spin devices.

A quantitative theoretical understanding of spin-dependent mesoscopic transport calls for developing efficient and flexible numerical algorithms, including matrix-reordering techniques within Green function approaches, which we will explain, review and employ.

\section{INTRODUCTION}

Charge and spin transport through phase-coherent conductors of mesoscopic scales carry imprints of wave interference as predominant and characteristic features: in the simplest case of a point contact, the conductance increases stepwise with Fermi energy, reflecting the discrete number of quantized open transverse channels contributing to transport; for more complex mesoscopic systems, such as ballistic quantum dots or diffusive conductors, the conductance typically wildly fluctuates, upon varying the Fermi energy or other parameters, around its classical mean value. Among the different effects on charge transport, the Aharonov-Bohm (AB) effect represents possibly the most genuine interference phenomenon at the heart of mesoscopic physics: The magnetoconductance of a ring conductor coupled to two leads exhibits distinct sinusoidal oscillations when monitored as a function of a perpendicular magnetic field threading the ring, with a period given by the magnetic flux quantum. As the AB signal stems from interfering waves travelling through the two different arms of the ring, it requires phase coherent wave functions extending over the ring typically on micron scales [1]. Hence the $\mathrm{AB}$ effect is frequently being used as a tool to investigate phase coherence and dephasing mechanisms of the orbital part of the wave functions, while the spin degree of freedom was usually neglected.

With rising interest in spin-dependent transport, the interplay of the electron spin and charge degree of freedom has been exploited in a variety of spin interference devices, to be discussed below. Different types of couplings to the electron spin have been considered for spin engineering in non-magnetic conductors. On the one hand this is possible through the Zeeman coupling to an externally applied magnetic field. Non-uniform $B$-fields with spatially varying direction are being employed to achieve a tailored spin dynamics, including the possibility for guided spin evolution or triggering spin flips. On the other hand, intrinsic spin-orbit (SO) interaction proves to be relevant in spin-dependent transport. It exists in systems with bulk inversion asymmetry and/or structure inversion asymmetry, e.g. due to the vertical confinement in semiconductor heterostructures (Rashba SO coupling [2, 3]).

Among the mesoscopic spin interference systems considered in the literature, ring geometries have again played 


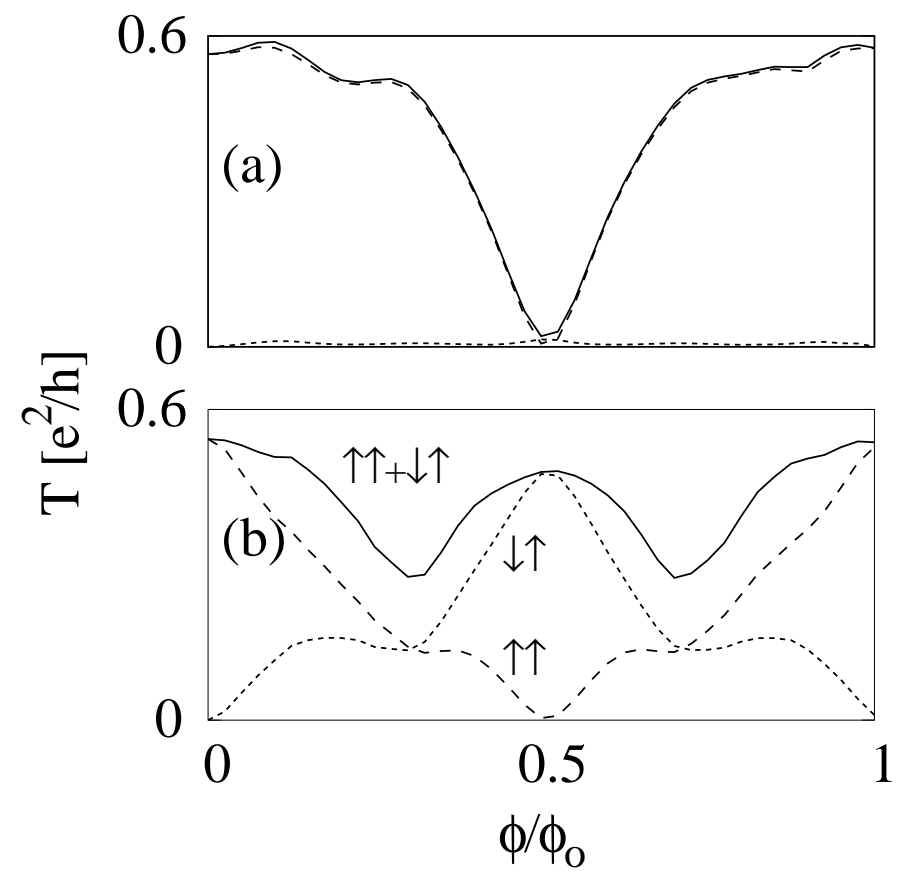

FIG. 2: Mesoscopic Aharonov-Bohm spin interference: The conductance of spin-up polarized carriers entering a single-channel two-dimensional Aharonov-Bohm ring (see Fig. 1) is shown as a function of flux $\phi=\pi r_{0}^{2} B$ (in units of the flux quantum $\phi_{0}=h c / e$ ) through the ring in the presence of Rashba spin-orbit coupling. For panel (a) and (b) the scaled spin-orbit coupling strength takes the values 0.2 and 1.0, expressed as the product $\omega_{R} T_{0} / 2 \pi$ of the precession frequency $\omega_{R}$ of the spin around the effective spin-orbit magnetic field and the time $T_{0}$ for travelling of the electrons around the ring. The overall conductance (solid line) is split into its components $G^{\uparrow \uparrow}$ (dashed line) and $G^{\downarrow \uparrow}$ (dotted line). Note in panel (b) the continuous change of the spin polarization, related to $G^{\uparrow \uparrow}-G^{\downarrow \uparrow}$, with $\phi$ and the spin switching at $\phi=\phi_{0} / 2$ (adapted from Ref. [4]).

an important role, opening up the field of spin-based AB physics (See Refs. [5, 6] for recent accounts including overviews over the literature). This includes topics such as Berry phase-signatures in transport, both theoretically [7, 8, 9, 10, 11, 12, 13] and experimentally [14, 15, 16, 17], spin-related conductance modulation [4, 18, 19], persistent currents 7, 20], spin Hall effect [21], spin filters 22, 23] and detectors 24], and spin switching mechanisms [5, 11].

To illustrate how the spin polarization can be tuned by exploiting (orbital and spin) interference in such an $A B$ setup, we consider as an introductory example spin switching in a two-dimensional (2D) ballistic phase-coherent ring symmetrically coupled to two single-channel leads (Fig. 1] Ref. [4]). We assume Rashba SO coupling which is relevant in conductors laterally defined on GaAs- or InAs-based two-dimensional electron gases (2DEGs). Rashba SO coupling will be defined and discussed in Sec. $\mathrm{V}$. It can be viewed as the coupling of the spin to a fictitious in-plane magnetic field directed perpendicular to the electron momentum. Hence in a ring it points mainly in radial direction. The strength of the SO field can be tuned by an external gate voltage [25] allowing to control experimentally the spin evolution.

We assume spin-polarized spin-up electrons entering the ring from the right (see Fig. 11). Fig. 2 displays numerically computed (see Sec. III) conductance traces as a function of the external magnetic flux $\phi$ for weak and moderate Rashba strengths. The overall conductance is presented as a solid line, and its spin-resolved components, $G^{\uparrow \uparrow}$ and $G^{\downarrow \uparrow}$, corresponding to outgoing spin-up and -down channels, are shown as dashed and dotted lines, respectively. In the weak SO coupling limit, Fig. 2(a), the overall conductance (solid line) shows the usual AB oscillations of period $\phi_{0}$ and is dominated by $G^{\uparrow \uparrow}$ (dashed line). As expected for weak spin-coupling, the spin polarization is almost conserved during transport. Interesting features appear for the case of moderate $\mathrm{SO}$ coupling depicted in panel (b). There, both components, $G^{\uparrow \uparrow}$ (dashed line) and $G^{\downarrow \uparrow}$ (dotted line), contribute similarly to the overall conductance (solid line). However, the spin polarization of the transmitted electrons varies as a function of the magnetic flux $\phi: G^{\downarrow \uparrow}=0$ at $\phi=0$, while $G^{\uparrow \uparrow}=0$ at $\phi=\phi_{0} / 2$. Hence, for zero flux all transmitted carriers conserve their original (incoming) spin-orientation, while for $\phi=\phi_{0} / 2$ the transmitted particles reverse their spin polarization. In other words: by tuning the magnetic flux from 0 to $\phi_{0} / 2$ we can reverse the spin-polarization of transmitted particles in a controlled way. Hence the $\mathrm{AB}$ ring with $\mathrm{SO}$ coupling acts as a tunable spin-switch. This switching can be traced back to constructive or destructive $\mathrm{AB}$ interference [5]. 
Switching a given spin polarization requires the generation of spin-polarized particles in non-magnetic mesoscopic conductors in the first place. Since spin injection from ferromagnets into a semiconductor remains problematic [26], alternative proposals have been made to achieve spin-polarized currents or spin accumulation without magnets, which we will briefly review in Sec. V] Among those are the spin Hall effect [27] and, in the context of coherent mesoscopic transport, concepts for Zeeman- and SO-mediated adiabatic spin pumping and spin ratchets.

For a recent account on spin phenomena in systems of reduced dimensions see 28]; for a review on the related field of magnetization dynamics and pumping in layered magnetic heterostructures see [29].

A complete and quantitative understanding of spin phenomena in the mesoscopic realm requires computational approaches to quantum transport which also serve as reference calculations for analytical predictions usually based on model assumptions. However, also numerical approaches cannot cope with the full many-body transport problem without relying on approximations. Here we focus on mesoscopic conductors, i.e. systems with a considerable number of electrons, with strong coupling to external leads. Then, a mean-field treatment is usually justified which allows one to reduce the Hamiltonian to a single-particle problem with an effective confinement potential resulting from a combination of external and mean-field potentials.

We further consider coherent transport close to equilibrium at relatively low bias, excluding inelastic effects, such that the Landauer approach to transport is justified. However, even in this case brute-force computational approaches quickly reach their limits: Conductors at mesoscopic scales are typically characterized by extensions which are (at least in one direction) much larger than the Fermi wavelength of the charge carriers, the shortest quantum scale involved. This implies rapidly oscillating, complex and often irregular spinor wave functions extending throughout the systems which require for the quantum mechanical numerical solution either huge sets of basis functions or, in tight-binding approaches, the use of rather fine, preferentially adapted grids. The strength of the wide-spread tight-binding approaches for transport discussed and reviewed here lies in their flexibility and general applicability. Moreover, tight-binding transport codes can be combined with density-functional (DFT) calculations for structure and electronic properties of the nano-conductors by using the parameters computed within DFT as input. This approach is frequently applied to transport in nano- or molecular electronics.

The paper is organized as follows: In the methodological Sec. III we will first briefly summarize and provide the key relations for spin quantum transport within the Landauer framework. In Sec. IV we focus on and explain in some detail advanced computational concepts, making use of graph-theory, to implement powerful and flexible algorithms for tight-binding transport codes. The numerical strength of the codes is demonstrated for ring-type geometries showing that by efficient tight-binding implementation one can gain orders of magnitude in performance. In Secs. V and $\nabla \mathrm{VI}$ we employ these numerical schemes to address two important aspects of spin-dependent transport, namely spin filtering and generating pure spin currents. To this end we focus on laterally-confined 2D ballistic nanostructures with Rashba SO interaction. We conclude with an outline of future research directions in mesoscopic spin transport which we consider important.

\section{NUMERICAL QUANTUM TRANSPORT}

\section{A. Landauer-Büttiker transport theory}

If the dimensions of a device get smaller than the phase coherence length $l_{\phi}$ of charge carriers, classical transport theories are not valid any more. Instead, carrier dynamics is now governed by quantum mechanics and the wave-like nature of particles becomes important. In general, the conductance/resistance of such a device does not follow Ohm's law.

Consider a two-point measurement setup as shown in Fig. 3; A scattering region is connected to large (phasebreaking) reservoirs by leads. The leads are assumed to be perfect and infinitely long to define asymptotic eigenstates $\phi_{n, \sigma}(y) e^{ \pm i k x}$ at energy $E$, where $n$ is the quantum number of transverse confinement - also called the channel number - and $\sigma$ is the spin index. The total scattering eigenstate $\psi_{n, \sigma}$ originating from channel $n$ with spin $\sigma$ in the left lead is, within the lead region, given by

$$
\psi_{n, \sigma}(\mathbf{x})=\left\{\begin{array}{ll}
\phi_{n, \sigma}(y) e^{i k x}+\sum_{m} r_{m, \sigma^{\prime} ; n, \sigma} \phi_{m, \sigma^{\prime}}(y) e^{-i k x} & \text { for } \mathbf{x} \text { in left lead } \\
\sum_{m} t_{m, \sigma^{\prime} ; n, \sigma} \phi_{m, \sigma^{\prime}}(y) e^{i k x} & \text { for } \mathbf{x} \text { in right lead }
\end{array},\right.
$$

and obeys the stationary Schrödinger equation $H \psi_{n, \sigma}=E \psi_{n, \sigma}$. The conductance $G$ in linear response can then be calculated within the Landauer-Büttiker formalism [30, 31, 32] (for tutorials see [1, 33]):

$$
G=\frac{e^{2}}{h} \sum_{n, m} \sum_{\sigma, \sigma^{\prime}} T_{m, \sigma^{\prime} ; n, \sigma}=\frac{e^{2}}{h} T_{\mathrm{C}}
$$




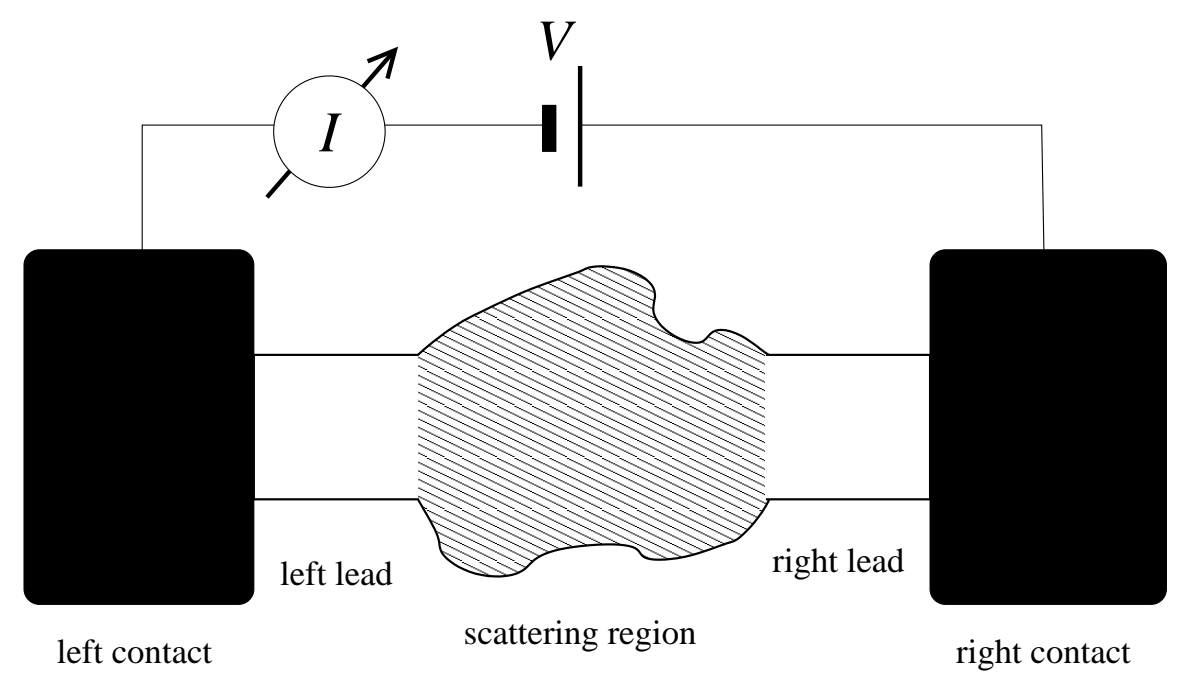

FIG. 3: Schematic view of a two-point measurement setup.

where $T_{m, \sigma^{\prime} ; n, \sigma}=\left|t_{m, \sigma^{\prime} ; n, \sigma}\right|^{2}$ is given by the squared transmission amplitudes of the scattering states $\psi_{n, \sigma}$. The fraction $\frac{e^{2}}{h}$ is called the conductance quantum. The scattering matrix $S_{m, \sigma^{\prime} ; n, \sigma}$ is a useful definition that combines reflection and transmission amplitudes for both leads into a single matrix. In this notation the index $n, \sigma$ then also contains information about the respective lead.

The problem of calculating the conductance $G$ is thus reduced to calculating the scattering eigenstates $\psi_{n, \sigma}$. Alternatively, the scattering amplitudes $r_{m, \sigma^{\prime} ; n, \sigma}$ and $t_{m, \sigma^{\prime} ; n, \sigma}$ can also be derived from the retarded Greens function $G^{\mathrm{R}}\left(\mathbf{x}, \mathbf{x}^{\prime}\right)$ of the system. The retarded Greens function for a given energy $E$ obeys the equation

$$
(E-H+i \eta) G^{\mathrm{R}}\left(\mathbf{x}, \mathbf{x}^{\prime}\right)=\delta\left(\mathbf{x}-\mathbf{x}^{\prime}\right),
$$

where $H$ is the Hamiltonian of the system and $\eta$ an infinitesimally small number. Formally this equation can be solved as

$$
G^{\mathrm{R}}=(E-H+i \eta)^{-1}
$$

The transmission and reflection amplitudes are then given by the Fisher-Lee relation [34]:

$$
\begin{gathered}
t_{m, \sigma^{\prime} ; n, \sigma}=-i \hbar \sqrt{v_{m} v_{n}} \int_{C_{\mathrm{R}}} d y \int_{C_{\mathrm{L}}} d y^{\prime} \phi_{m, \sigma^{\prime}}(y) G^{\mathrm{R}}\left(\mathbf{x}, \mathbf{x}^{\prime}\right) \phi_{n, \sigma}\left(y^{\prime}\right), \\
r_{m, \sigma^{\prime} ; n, \sigma}=\delta_{m n} \delta_{\sigma^{\prime} \sigma}-i \hbar \sqrt{v_{m} v_{n}} \int_{C_{\mathrm{L}}} d y \int_{C_{\mathrm{L}}} d y^{\prime} \phi_{m, \sigma^{\prime}}(y) G^{\mathrm{R}}\left(\mathbf{x}, \mathbf{x}^{\prime}\right) \phi_{n, \sigma}\left(y^{\prime}\right),
\end{gathered}
$$

where $v_{n}$ denotes the velocity of channel $n$ and the integration runs over the cross-section $C_{\mathrm{L}}\left(C_{\mathrm{R}}\right)$ of the left (right) lead. Equations (5) and (6) are valid only for leads without magnetic fields and no spin-orbit interaction. Baranger and Stone [35] have extended the formalism to also account for arbitrary magnetic fields in the leads, and their description can also be applied to finite spin-orbit interaction.

\section{B. Tight-binding representation of the Hamiltonian}

Except for very simple geometries, the scattering problem cannot be solved analytically. Therefore, the use of computers for a numerical solution of the scattering problem is very often the method of choice. However, the related stationary Schrödinger equation $H \psi=E \psi$ is a differential equation with continuous degrees of freedom that are difficult to treat on a computer. In general, a numerical solution is thus only attempted within a discrete basis set which converts the differential equation into a matrix equation. 


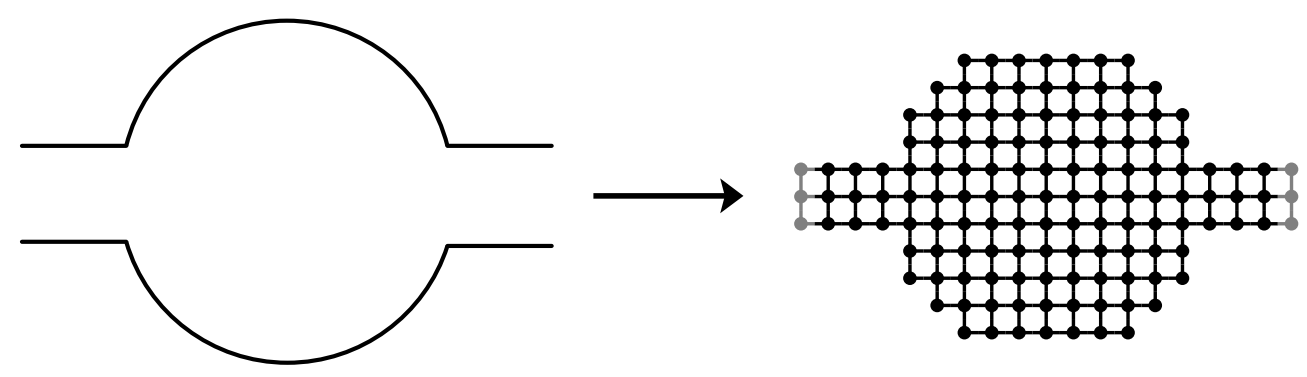

FIG. 4: Discretizing a continuous region on a square grid.

The method of finite differences is a very simple and yet powerful way to introduce such a discrete basis set and has been applied to the Schrödinger equation already as early as 1934 [36, 37] (for an introduction see [1, 33]). Here we illustrate, as an example, the application of the method for a simple one-dimensional effective mass Hamiltonian including a potential

$$
H=-\frac{\hbar^{2}}{2 m} \frac{d^{2}}{d x^{2}}+V(x) .
$$

In the method of finite differences, space is approximated by a grid of discrete lattice points spaced equidistantly with lattice constant $a$. For the 2D case, usually a square grid is used as depicted in Fig. (4). Using the Taylor expansion of the wave function $\psi$ we can write

$$
\begin{aligned}
& \psi(x+a)=\psi(x)+\psi^{\prime}(x) a+\frac{1}{2} \psi^{\prime \prime}(x) a^{2}+\frac{1}{6} \psi^{(3)}(x) a^{3}+\frac{1}{24} \psi^{(4)}(x) a^{4}+\ldots \\
& \psi(x-a)=\psi(x)-\psi^{\prime}(x) a+\frac{1}{2} \psi^{\prime \prime}(x) a^{2}-\frac{1}{6} \psi^{(3)}(x) a^{3}+\frac{1}{24} \psi^{(4)}(x) a^{4}+\ldots .
\end{aligned}
$$

Adding Eqs. (8) and (9), we arrive at an expression for the second derivative of the wave function in terms of values of the wave function on the grid,

$$
\frac{d^{2}}{d x^{2}} \psi(x)=\frac{1}{a^{2}}(\psi(x+a)+\psi(x-a)-2 \psi(x))+\mathcal{O}\left(a^{2}\right),
$$

valid up to second order in the lattice spacing $a$. The differential equation

$$
-\frac{\hbar^{2}}{2 m} \frac{d^{2}}{d x^{2}} \psi(x)+V(x) \psi(x)=E \psi(x)
$$

is thus replaced by a set of difference equations

$$
-\frac{\hbar^{2}}{2 m a^{2}}\left(\psi\left(x_{i+1}\right)+\psi\left(x_{i-1}\right)-2 \psi\left(x_{i}\right)\right)+V(x) \psi\left(x_{i}\right)=E \psi\left(x_{i}\right)
$$

where $x_{i \pm 1}=x_{i} \pm a$, yielding the tight-binding representation of the Hamiltonian:

$$
H=\sum_{x_{i}}-\frac{\hbar^{2}}{2 m a^{2}}\left(\left|x_{i}\right\rangle\left\langle x_{i+1}\right|+\text { h.c. }\right)+\left(2 \frac{\hbar^{2}}{2 m a^{2}}+V\left(x_{i}\right)\right)\left|x_{i}\right\rangle\left\langle x_{i}\right| .
$$

Here, $\left|x_{i}\right\rangle$ denotes a state localized at grid point $x_{i}$.

In principle, the quality of the finite differences approximation can be improved up to a desired precision by reducing the lattice spacing $a$. However, since this leads to a larger problem size, the minimum lattice spacing achievable is set by the available computing time and memory. Thus, one must keep an eye on the validity of the finite differences approximation. In Fig. [5] we show the energy spectrum $E(k)$ for the continuous one-dimensional Schrödinger equation and the tight-binding (finite differences) approximation. The tight-binding approximation only holds for $k a \ll 1$ and $E \ll \frac{\hbar^{2}}{2 m a^{2}}$, and it does not make sense to consider the whole energy spectrum given by the tight-binding band width. The method of finite differences presented here can straight-forwardly be applied to more complex Hamiltonians, 


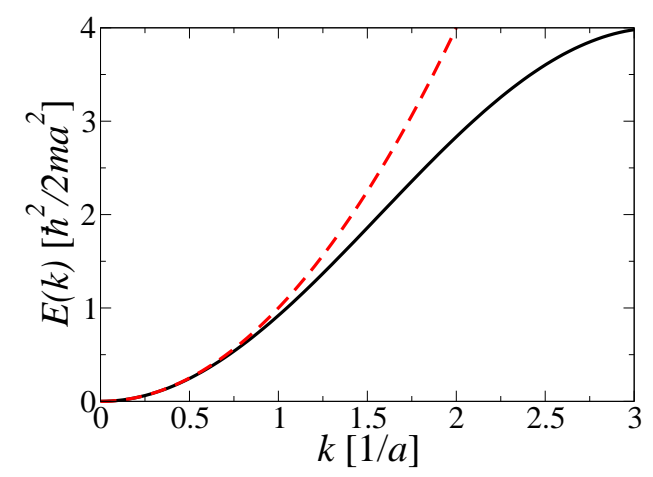

FIG. 5: Energy spectrum $E(k)$ for the continuous Schrödinger equation (red dashed line) and the tight-binding approximation (black solid line)

including for example spin-orbit interactions [4], and will be later used to calculate transport phenomena including spin in sections $\mathrm{V}$ and $\mathrm{VI}$

A tight-binding representation of the Hamiltonian can also be obtained by applying the finite element method [38]. Furthermore tight-binding Hamiltonians are also used in treatments beyond the effective mass approximation, such as from atomic orbitals in empirical tight-binding models [39, 40, 41], or from orbitals of the Kohn-Sham equations within DFT [42, 43, 44].

\section{Numerical algorithms}

Within the tight-binding approximation the Hamiltonian $H$ can be represented by a matrix, even though this matrix is still infinite as the leads are infinitely long. However, the infinite matrix problem can be reduced to a finite problem by partitioning the system into three isolated parts: left lead, scattering region, and right lead. The Hamiltonian then reads

$$
H=\left(\begin{array}{ccc}
H_{\mathrm{L}} & V_{\mathrm{LS}} & 0 \\
V_{\mathrm{SL}} & H_{\mathrm{S}} & V_{\mathrm{SR}} \\
0 & V_{\mathrm{RS}} & H_{\mathrm{R}}
\end{array}\right),
$$

where $H_{\mathrm{L}(\mathrm{R})}$ is the (infinite) Hamiltonian of the left (right) lead, $H_{S}$ is the Hamiltonian of the scattering region and of finite size. Since the leads are always chosen such that asymptotic eigenstates can be defined, the Hamiltonian of the isolated leads must contain some periodicity that facilitates calculating their Greens functions $g_{\mathrm{L}, \mathrm{R}}^{\mathrm{R}}$. This can be done analytically for simple systems [1, 33], for more complex situations the Greens function can be calculated numerically either by iteration [45, 46] or semianalytical formulas [40, 44, 47]. Introducing the retarded self-energy $\Sigma^{\mathrm{R}}=\sum_{i=L, R} V_{\mathrm{S} i} g_{i}^{\mathrm{R}} V_{i \mathrm{~S}},\left[1,33\right.$ ] the Greens function $G_{\mathrm{S}}$ of the scattering region is given by

$$
G_{\mathrm{S}}^{\mathrm{R}}=\left(E-H-\Sigma^{\mathrm{R}}\right)^{-1}
$$

reminiscent of Eq. (4) but with an effective Hamiltonian $H+\Sigma^{\mathrm{R}}$.

The original infinite-dimensional problem has thus been reduced to a finite size matrix problem that can, in principle, be solved straight-forwardly on a computer. However, for any but rather small problems, the computational task of the direct inversion in Eq. (15) is prohibitive. Therefore, many algorithms make use of the sparsity of the Hamiltonian matrix in tight-binding representation - in particular they employ the property that this matrix can be written in 


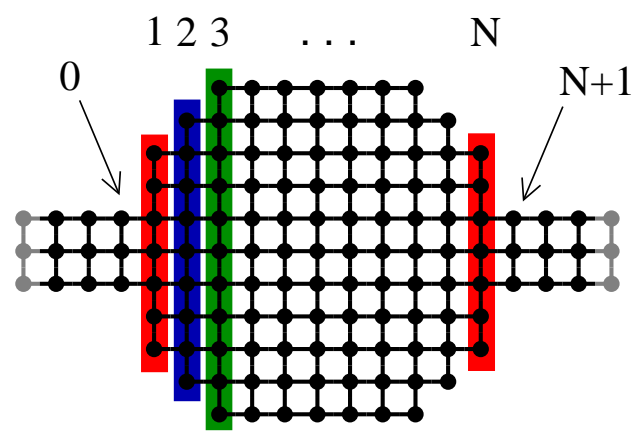

FIG. 6: Block-tridiagonal matrix form arising in the method of finite differences. Grid points with the same $x$-coordinate are placed into the same block.

block-tridiagonal form

$$
H=\left(\begin{array}{cccccccccc}
\ddots & & & & & & & & & \\
& H_{\mathrm{L}} & V_{\mathrm{L}} & & & & & & & \\
& V_{\mathrm{L}}^{\dagger} & H_{\mathrm{L}} & H_{01} & & & \ddots & & & \\
& & H_{10} & H_{11} & H_{12} & & & 0 & & \\
& & H_{21} & H_{22} & H_{23} & & & \ddots & \\
& & & H_{32} & \ddots & & & & \\
& & & & & \ddots & H_{N-1 N} & & \\
& & 0 & & & H_{N N-1} & H_{N N} & H_{N N+1} & \\
& & & \ddots & & & H_{N+1 N} & H_{\mathrm{R}} & V_{\mathrm{R}} & \\
& & & & & & & V_{\mathrm{R}}^{\dagger} & H_{\mathrm{R}} & \\
& & & & & & & & & \ddots
\end{array}\right) .
$$

Here the index $\mathrm{L}(\mathrm{R})$ denotes the blocks in the left (right) lead, $1 \ldots N$ the blocks within the scattering region, and 0 , $(N+1)$ the first block in the left (right) lead. Such a form arises naturally in the method of finite differences, when grid points are grouped into vertical slices according to their $x$-coordinates, as shown in Fig. 6. but also applies to any other sparse tight-binding Hamiltonians.

The block-tridiagonal form of the Hamiltonian is the foundation of several quantum transport algorithms, together with the fact that, according to Eqs. (5) and (6), only the blocks $G_{N+10}^{\mathrm{R}}$ and $G_{00}^{\mathrm{R}}$ of the Greens function $G^{\mathrm{R}}$ are needed for the calculation of transmission and reflection probabilities. The transfer matrix approach applies naturally to block-tridiagonal Hamiltonians, but becomes unstable for larger systems. However, a stabilized version has been developed by Usuki et al. [48, 49]. In the decimation technique [50, 51], the Hamiltonian of the scattering region is replaced by an effective Hamiltonian between the two leads by eliminating internal degrees of freedom. The contact block reduction method [52] calculates the full Greens function of the system using a limited set of eigenstates. The recursive Greens function (RGF) technique [53, 54, 55] uses Dyson's equation to build up the system's Greens function block by block. It has also been adapted to Hall geometries with four terminals [56] and to calculate non-equilibrium densities [57, 58]. Furthermore, the RGF algorithm has been formulated to be suitable for parallel computing [59], and the modular recursive Greens function (MRGF) method is an extension to take advantage of special geometries, such as circles or rectangles [60, 61].

Here we give a simple form of the RGF algorithm as described in [33, 55]. The RGF method makes use of Dyson's equation

$$
G=G_{0}+G_{0} V G
$$

to add successively blocks to the system, as depicted in Fig. 7 . Let $G^{\mathrm{R},(i)}$ denote the Greens function for the system containing all blocks $\geq i$. Then, at energy $E$, the Greens function $G^{\mathrm{R},(i)}$ is related to $G^{\mathrm{R},(i+1)}$ by

$$
G_{i i}^{\mathrm{R},(i)}=\left(E-H_{i i}-H_{i i+1} G_{i+1 i+1}^{\mathrm{R},(i+1)} H_{i+1}\right)^{-1} \text { and }
$$




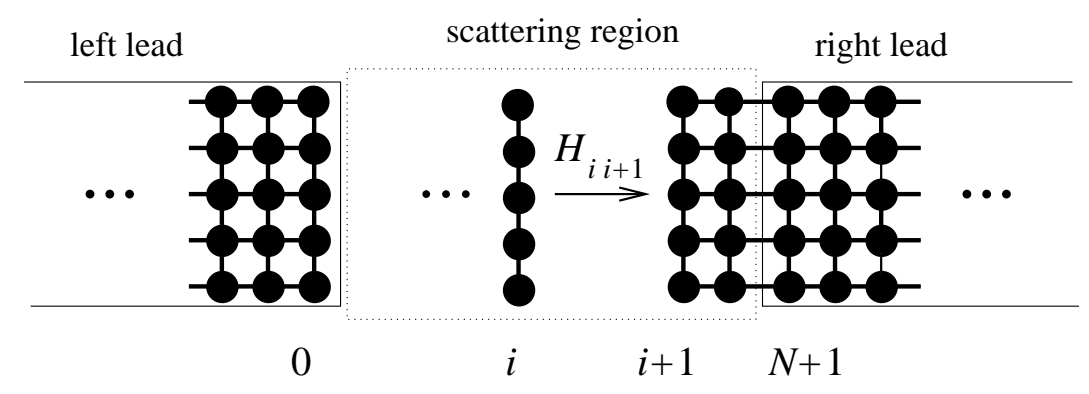

FIG. 7: Schematic view of the recursive Greens function algorithm: the system is built up adding block by block.

$$
G_{N+1 i}^{\mathrm{R},(i)}=G_{N+1 i+1}^{\mathrm{R},(i+1)} H_{i+1 i} G_{i i}^{\mathrm{R},(i)} .
$$

Starting from $G_{N+1 N+1}^{\mathrm{R},(N+1)}=g_{\mathrm{R}}^{\mathrm{R}}$, the surface Greens function of the right lead, $N$ slices are added recursively, until $G^{\mathrm{R},(1)}$ has been calculated. The blocks of the Greens function of the full system necessary for transport are then given by

$$
\begin{gathered}
G_{00}^{\mathrm{R}}=\left(\left(g_{\mathrm{L}}^{\mathrm{R}}\right)^{-1}-H_{01} G_{11}^{\mathrm{R},(1)} H_{10}\right)^{-1} \text { and } \\
G_{N+10}^{\mathrm{R}}=G_{N+11}^{\mathrm{R},(1)} H_{10} G_{00}^{\mathrm{R}},
\end{gathered}
$$

where $g_{\mathrm{L}}^{\mathrm{R}}$ is the surface Greens function of the left lead.

Each step of the algorithm performs inversions and matrix multiplications with matrices of size $M_{i}$. Since the computational complexity of matrix inversion and multiplications scales as $M_{i}^{3}$, the complexity of the RGF algorithm is $\propto \sum_{i=0}^{N+1} M_{i}^{3}$. Thus, it scales linearly with the "length" $\mathrm{N}$, and cubically with the "width" $M_{i}$ of the system.

While for certain geometries the RGF algorithm cannot compete with more specialized algorithms such as MRGF, it is very versatile and easily adapted to many situations, and is thus our method of choice. In the next section we will discuss matrix reordering techniques that improve the runtime of the RGF algorithm considerably and allow the treatment of arbitrary systems.

\section{MATRIX REORDERING STRATEGIES FOR QUANTUM TRANSPORT}

\section{A. Graph-theoretical approaches to matrix reordering}

As shown in the previous section, the structure of a Hamiltonian matrix $H$ does influence the runtime of the RGF algorithm. Thus, the runtime of the algorithm can potentially be improved by reordering the matrix with a permutation $P$,

$$
H^{\prime}=P H P^{-1} \text {. }
$$

At first glance, such an effort may seem pointless: For example, the block-tridiagonal structure naturally associated with a finite difference grid (as discussed in section III) leads to a sparse matrix with a small bandwidth, as shown in Fig. 8. However, as we show below, the choice of a suitable permutation $P$ can still lead to a significant speed-up of the RGF algorithm.

For this we define a weight $w(H)$ associated with a given matrix $H$ as

$$
w(H)=\sum_{i=0}^{N+1} M_{i}^{3} \quad \text { where } M_{i} \text { is the size of block } H_{i i} .
$$

Optimizing the matrix for the RGF algorithm is then equivalent to minimizing the weight $w(H)$. Since $\sum_{i=0}^{N+1} M_{i}=$ $N_{\text {grid }}$, where $N_{\text {grid }}$ is the total number of grid points, $w(H)$ is minimal, if all $M_{i}$ are equal, and $M_{i}=N_{\text {grid }} /(N+2)$. Therefore, a matrix tends to have small weight, if the number $N$ of blocks is large, and all blocks are equally sized. The reordering problem of the matrix $H$ is thus summarized as follows:

Matrix reordering problem: Find a reordered matrix $H^{\prime}$ such, that 

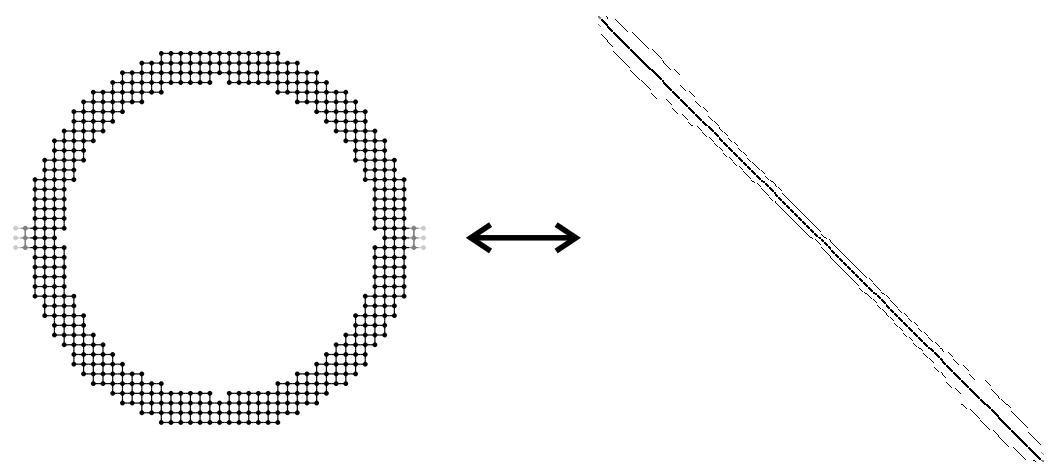

FIG. 8: Example of a sparsity structure of a matrix associated with a finite difference grid. Black dots mark non-zero entries. The picture of the finite difference grid can also be interpreted as a graphical representation of a graph.

1. $H_{00}^{\prime}$ and $H_{N+1 N+1}^{\prime}$ are blocks given by the left and right leads (as required by the RGF algorithm),

2. $H^{\prime}$ is block-tridiagonal $\left(H_{i j}^{\prime} \neq 0\right.$, iff $\left.j=i+1, i, i-1\right)$,

3. the number $N$ of blocks is as large as possible, and all blocks are equally sized.

These requirements define the optimization problem of reordering the matrix $H$. Usually, in such optimization problems finding the best solution deterministically is prohibitively expensive, and one has to resort to heuristic strategies.

In order to do that, we reformulate our matrix problem using concepts from graph theory. A graph $G$ is a pair $G=(V, E)$, where $V$ is a set of vertices $i$, and $E$ a set of pairs of vertices $(i, j) \in V \times V$. Such a pair is called an edge. A graph is called undirected, if for every edge $(i, j) \in E$ also $(j, i) \in E$. Two vertices $i$ and $j$ are called adjacent, if $(i, j) \in E$. A graph can be visualized by drawing dots for each vertex $i$ and lines connecting these dots for every edge $(i, j)$. It should be noted, that all the pictures of finite difference grids shown so far can directly be interpreted as graphs. There is a natural one-to-one correspondence between graphs and the structure of sparse matrices. For a given $n \times n$ matrix $H$, we define a graph $G=(V, E)$ with $V=\{1, \ldots, n\}$ and $(i, j) \in E$ iff $H_{i j} \neq 0$. Thus, the symmetric zero-nonzero structure of Hermitian matrices, as considered in quantum transport, leads to associated undirected graphs. An example of such a correspondence between a graph and a matrix has already been shown in Fig. 8. With respect to matrices, graphs are also very convenient for storing and handling sparse matrix data structures on a computer.

In terms of graph theory, matrix reordering corresponds to renumbering the vertices of a graph. Since we are only interested in reordering the matrix in terms of matrix blocks (the order within a block should not matter too much), we define a partitioning of $G$ as a set $\left\{V_{i}\right\}$ of disjoint subsets $V_{i} \subset V$ such that $\bigcup_{i} V_{i}=V$ and $V_{i} \cap V_{j}=\emptyset$ for $i \neq j$. Using these concepts, we can now reformulate the original matrix reordering problem into a graph partitioning problem:

Graph partitioning problem: Find a partitioning $\left\{V_{0}, \ldots, V_{N+1}\right\}$ of $G$ such that:

1. $V_{0}$ and $V_{N+1}$ contain the vertices belonging to left and right leads,

2. there are edges between $V_{i}$ and $V_{j}$ iff $j=i+1, i, i-1$ (block-tridiagonality),

3. the number of sets $V_{i}$ is as large as possible and all sets $V_{i}$ have the same cardinality. Such a partitioning with all $V_{i}$ equally sized is called balanced.

A partitioning that meets requirement 2 is called a level set with levels $V_{i}$ and appears commonly as an intermediate step in algorithms for bandwidth reduction of a matrix [62, 63, 64, 65]. These algorithms seek to find a level set of minimal width, i. e. $\max _{i=0 \ldots N+1}\left|V_{i}\right|$ as small as possible which is equivalent to requirement 3 . The major difference between our graph partitioning problem and the bandwidth reduction algorithms is requirement 1: In our case the start and end blocks are given by the geometry of the system, whereas in the bandwidth reduction methods these can be chosen freely. The implications of this difference will be discussed below.

The bandwidth reduction algorithms start with the observation that a breadth first search (BFS) starting from any vertex in the graph creates a level set: In our situation, the BFS starts from level $V_{0}$ of the left lead. Then, successively for $i=0,1,2, \ldots$, all vertices adjacent to $V_{i}$ that have not been assigned to a level yet are placed in $V_{i+1}$. This construction ensures that each level $V_{i}$ only has edges connecting to vertices in $V_{i+1, i, i-1}$ and thus ensures 
block-tridiagonality. The search stops, once a vertex adjacent to the right lead is encountered, and all unassigned vertices are placed into the last level $V_{N}$. The number of BFS steps determines therefore the maximum number $N$ of blocks and is related to the minimum distance between left and right lead in the graph. However, this construction of the level set also suffers from a serious problem: Depending on the distance between the leads, the last level $V_{N}$ can potentially contain a large number of vertices leading to a very unbalanced partitioning. In the bandwidth reduction methods, the first and the last vertex are chosen to have (to a good approximation) maximum distance, and thus this problem does not occur there. Hence, conventional bandwidth reduction algorithms can only be applied to quantum transport problems, if the leads are - in terms of the underlying graph - furthest apart.

In this study, we will consider two reordering algorithms: First, the Gibbs-Poole-Stockmeyer (GPS) algorithm 65], a widely used bandwidth reduction algorithm. The GPS algorithm combines the level sets originating from a BFS from both left and right lead to give an optimized level set. Due to the limitations discussed above, it can only be used efficiently for systems with leads far apart. To overcome this difficulty partly, we also propose a second algorithm, later referred to as BFS partitioning: The system is bisected recursively by means of a simultaneous BFS from left and right leads. In a bisection process, vertices that are closer (as given by the number of BFS steps) to the left (right) lead are placed into the left (right) level. The resulting two levels are then further bisected recursively until the final level set has been constructed. This algorithm tries to avoid an unbalanced partitioning, as every step tries to create a balanced bisection. We have found this global approach — as opposed to the local approach in the BFS - to yield balanced partitionings for systems where there are only few local minima in the weight $w(H)$. For general systems, a more sophisticated method should be used [66].

Obviously, reordering the matrix will only improve the runtime of the RGF algorithm, if the overhead of the reordering process is small compared to the actual transport calculation. Because of this reason, applying general optimization algorithms to the matrix reordering problem is not an option. Instead, heuristics designed for graph problems give much better performance. The GPS algorithm scales linearly with the number of edges $|E|$, and since in a tight-binding representation $|E| \propto N_{\text {grid }}$, its algorithmic complexity is $\mathcal{O}\left(N_{\text {grid }}\right)$, whereas the BFS partitioning algorithm scales as $\mathcal{O}\left(N_{\text {grid }} \log N_{\text {grid }}\right)$. In any case, the scaling is much more favorable than that of the RGF algorithm, $\propto \sum_{i=0}^{N+1} M_{i}^{3}$, so that for systems of typical size the overhead of the reordering process becomes negligible, as we will demonstrate in the next section.

\section{B. Example: ring geometry}

In order to demonstrate the performance of the algorithms discussed above, we consider their application to a ring geometry in finite difference approximation. The performance of the RGF algorithm after matrix reordering is compared with the performance using the ordering that arises naturally in finite difference grids as shown in Fig. 6 (in the remainder referred to as natural partitioning).

In Fig. 9(a)-(d) we show four different approaches for calculations in a ring geometry. A ring can be treated as a circular cavity (see Fig. 9(a)), with a large potential on the lattice points inside the inner ring diameter. This approach is easier to implement than a real ring but less efficient, as a large additional number of lattice points enters the calculation. However, this approach has been used frequently in the past, and therefore we also consider its performance. Transport calculations in a real ring require somewhat more bookkeeping because of the nontrivial geometry, but can be easily done describing the grid as a graph. For the circular cavity, we only consider natural partitioning, as shown in Fig. 9(a), for the ring we consider natural partitioning (Fig. 9(b)), GPS partitioning (Fig. 9(c)) and BFS partitioning (Fig. 9(d)).

The partitionings in Fig. 9)(c) and (d) are dramatically different from the natural partitioning. The levels align mainly in vertical or diagonal directions as these are the preferred directions in the square lattice. The number of levels is increased with respect to the natural partitioning, as the distance between both leads is much larger than the number of vertical slices, leading to a larger number of blocks in the block-tridiagonal matrix, as can be seen from Fig. 9(e). Both GPS and BFS partitioning lead to a drastically reduced block size with respect to the natural partitioning and the result is rather balanced. Though the actual partitionings in Fig. 9)(c) and (d) look quite different, with respect to minimizing the weight $w(H)$ they are equally good. The BFS partitioner conforms to the geometric structure, as it puts vertices in levels according to their distance from the leads. Except for a small number of blocks in the beginning and the end of the block-tridiagonal matrix (see Fig. 9(e)), the GPS partitioner leads to an equally balanced structure, although the partitioning looks quite different. The GPS partitioner works well in this case, as the leads are almost at maximum distance in this ring structure.

We now apply the recursive Greens function algorithm from Sec. III to the different partitionings. In Fig. 10(a) we show the runtime of the algorithm as a function of the number of lattice points in the leads, which is also the number of lattice points across the arms of the ring. Note that in all cases the runtime includes both the time spent in calculating the matrix reordering and the time spent in the actual transport calculation. 
a)

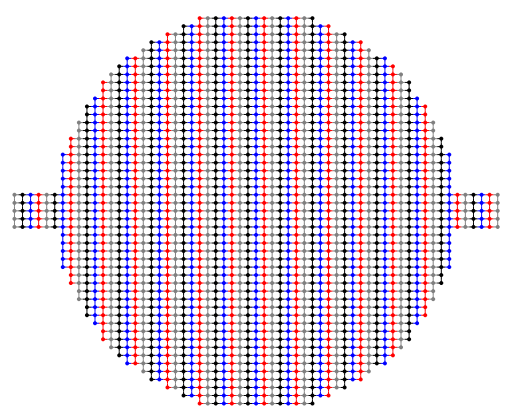

d)

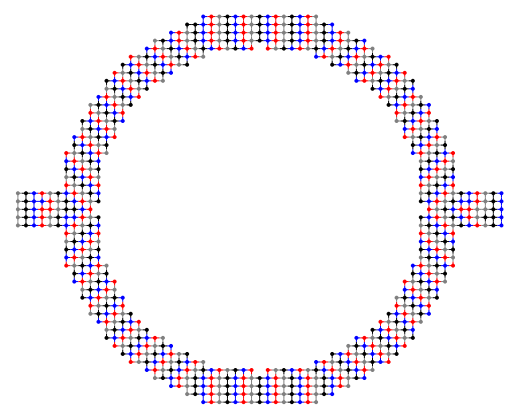

b)

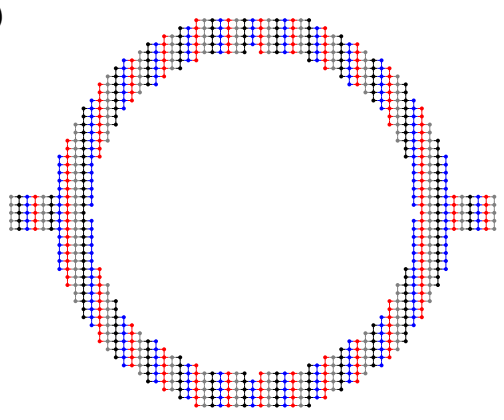

c)

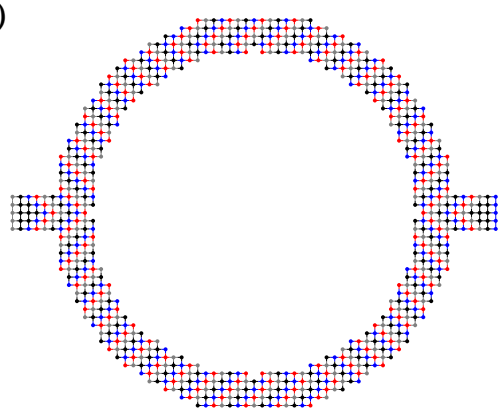

e)

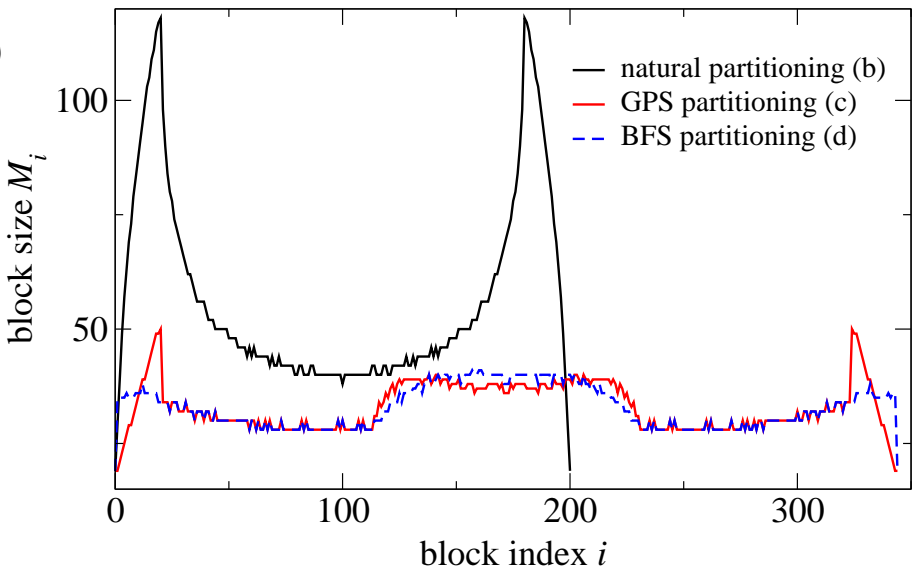

FIG. 9: Partitioning of the different systems considered in the performance study. (a) Circular cavity in natural partitioning, where the ring structure is enforced by a potential term in the middle of the cavity, (b) ring in natural partitioning, (c) ring in GPS partitioning and (d) ring in BFS partitioning. In (a)-(d), vertices belonging to the same level in the partitioning are marked with the same color, different levels are marked in alternating colors. Note that, in order to reveal the partitioning structure more clearly, the grids shown here are much smaller than in a typical calculation. (e) Size $M_{i}$ of the blocks $H_{i i}$ in the block-tridiagonal matrix for a ring with 20 lattice points in the leads for natural, GPS and BFS partitioning (the circular cavity is not shown here).

The runtime scales similarly in all cases, as this is the scaling of the RGF algorithm. Nevertheless, the runtimes of the different approaches can differ by a factor that is significant. As expected, the circular cavity is slowest, due to the extra number of lattice points. GPS and BFS partitionings lead to a rather similar performance that is significantly better than the ring in natural partitioning. It outperforms the circular cavity even by a factor of up to 100 . In the remainder, we examine the performances of the ring for different partitionings in more detail and leave out the circular cavity. In Fig. 10(b) we show the relative performance gain of GPS and BFS partitionings over the natural partitioning. Except for the smallest of systems that are too small to be useful in practice, the performance of GPS and BFS partitionings is better than the natural partitionings. Even for moderately sized systems the performance gain through the matrix reordering is approximately 3 , with the BFS partitioner being slightly better than the GPS partitioner. In Fig. 10(b) we also show estimates of the performance gain calculated from the weights $w(H)$ of the different partitionings. These estimates predict a performance gain of approximately 4 . For small system sizes, we see deviations from these estimates because of the overhead of the partitioning process, for larger system sizes we almost reach the estimated value. On modern computer architectures, runtime does not only depend on the number of arithmetic operations [67], and thus we do not achieve the full theoretical potential of the reordering, yet still significant improvements. In Fig. 10(c) we show the fraction of time spent in calculating the matrix reordering with respect to the total computation time, and as expected the overhead becomes negligible already for moderately sized systems. It should be noted that in actual calculations the partitioning is commonly only done once, and transport calculations can be done repeatedly with the same partitioning: Usually one is interested in transport properties depending on some parameters, and these generally do not change the structure of the Hamiltonian matrix but only the values of the respective entries. In this case, the partitioning overhead becomes even more irrelevant.

It should be emphasized, that for all the situations, the same transport code was used. In addition to the significant 

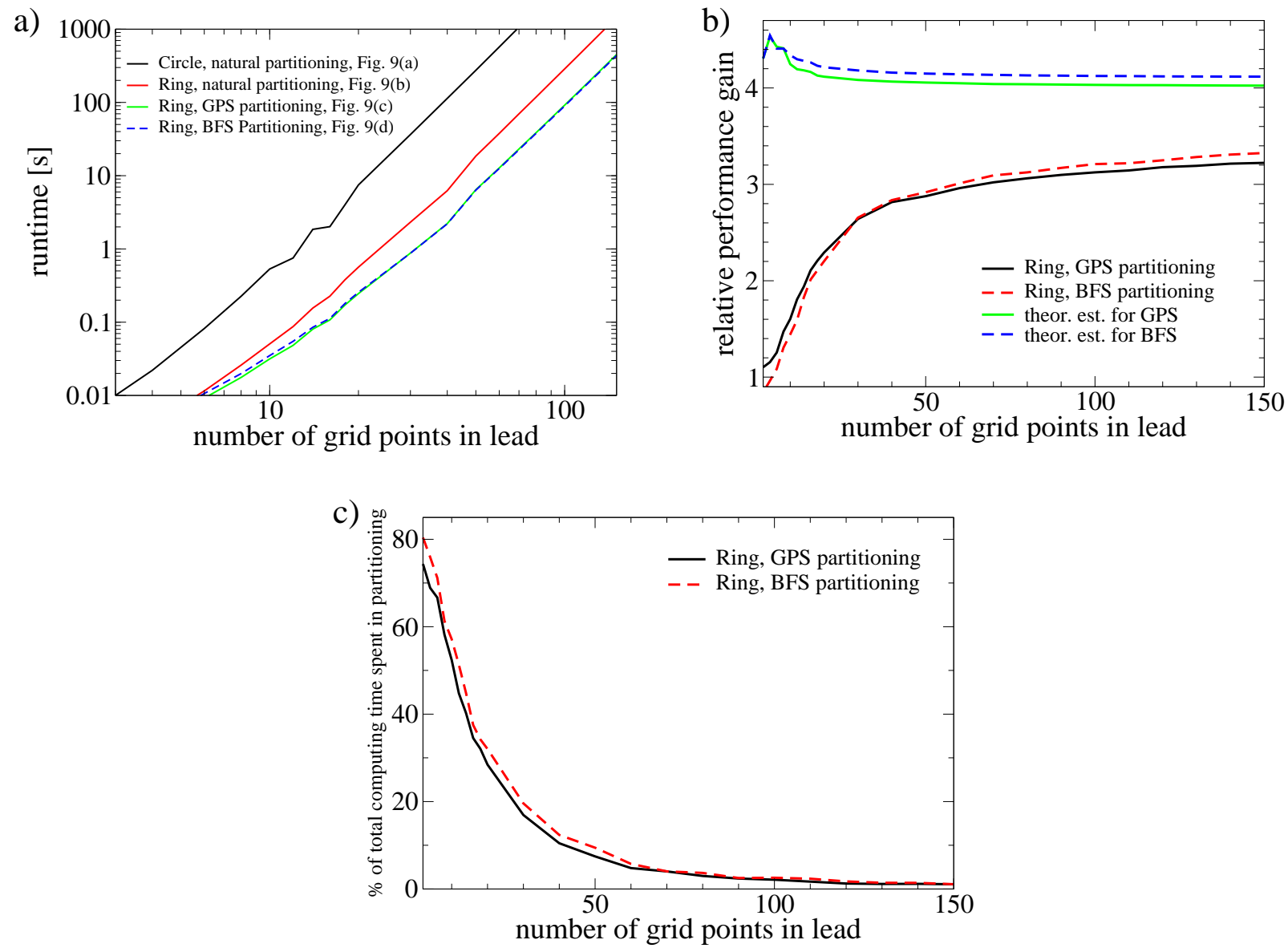

FIG. 10: Performance of the partitionings (for rings, Fig. 9): (a) runtime of the different systems as a function of the number of lattice points in the leads, i.e. as a function of system size. The calculations were performed on a Core2Duo T5500 processor and 1GB of memory, and the runtime includes both partitioning overhead and transport calculation. (b) Performance gain of GPS and BFS partitionings with respect to natural partitioning and (c) overhead of the matrix reordering as a function of system size.

speedup through the graph techniques considered here, the abstraction of the system through graph structures allows for very generic transport codes. This is an additional strength of this approach, as the well-established RGF algorithm is thus readily applied to arbitrary systems that would require special treatment otherwise, such as a scattering region with perpendicular leads, as depicted in Fig. 11]

Of course, for any system, an algorithm designed for that special system will probably outperform generic approaches. However, the combination of matrix reordering and RGF algorithm can be applied to arbitrary systems and is thus probably the most versatile transport approach. In addition, all the algorithms relying on the blocktridiagonal structure of the Hamiltonian mentioned in Sec. III can benefit from these matrix reordering strategies.

Modern transport calculations tend to be more and more complex and time-consuming. For example, an electronic calculation including the electron spin typically takes $2^{3}=8$ times longer than a calculation on spin-less electrons. Furthermore, calculations including disorder involve averages over many disorder configurations. Any increase in computation speed is therefore beneficial, and the added versatility through matrix reordering methods makes these techniques even more useful.

\section{SPIN FILTERING IN NANOSTRUCTURES}

In the introductory example in Sec. II it was shown how to realize systems that work as spin switches making use of the interference of wavefunctions propagating clockwise and counterclockwise in Aharonov-Bohm rings with SO-interaction. However, several other device proposals have been put forward making use of different concepts in 


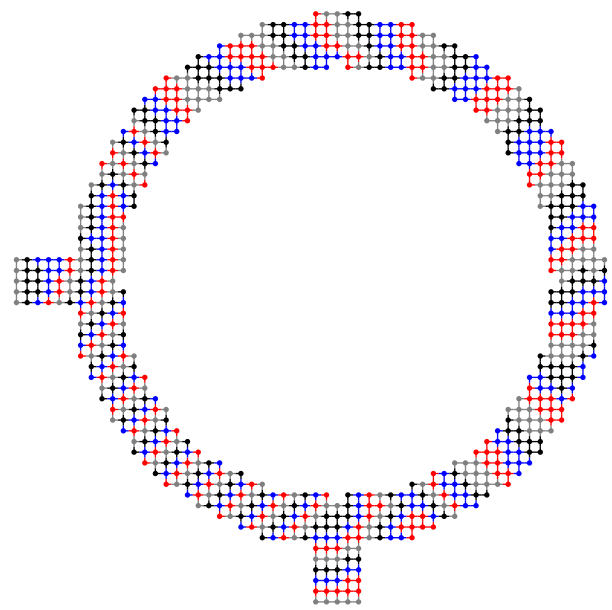

FIG. 11: BFS partitioning of a ring with perpendicular leads. Note that in this case the two leads are closer together and thus the number of blocks is reduced. Therefore the block sizes tend to be larger than in the previous examples.

order to achieve spin filtering in mesoscopic systems.

A very prominent category is transverse focusing of ballistic electrons/holes in two dimensional electron/hole gases (2DEGs/2DHGs) [68]. In materials that exhibit SO interaction the cyclotron radius of ballistic electrons/holes due to a magnetic field perpendicular to the $2 \mathrm{DEG} / 2 \mathrm{DHG}$ depends on the spin state of the charge carriers. Therefore, it is possible to filter out either spin-up or spin-down electrons by an appropriate arrangement of quantum point contacts and a proper choice of the perpendicular magnetic field. Apart from several theoretical treatments of this topic [69, 70, 71], spin filtering by transverse focusing has already been experimentally verified in a GaAs-based 2DHG. 72] In related experiments, spin-polarized currents in 2DEGs/2DHGs were detected by a setup consisting of point contacts and making use of transverse focusing of the charge carriers 73 . With such a detector it was possible to confirm the presence of spin-polarized currents emitted from mesoscopic quantum dots utilizing quantum interference at high in-plane magnetic fields [74] and from quantum point contacts, which were either made spin sensitive with high in-plane magnetic fields [73] or showed a pronounced "0.7-anomaly" [75].

A further appealing approach to filter spins are three terminal structures, that act as mesoscopic Stern-Gerlach type spin filters [6]. In these devices one of the leads injects spin-unpolarized current and, after passing a region where the spin-degeneracy is lifted, oppositely-polarized output currents exit through the other two leads. This separation of up and down spins can be accomplished, e.g., by utilizing Rashba SO interaction [77, 78, 79].

However, three terminals are not required to create spin polarized currents. Many devices, as e.g. the AB-ring presented in Sec. II typically rely on two terminals only, where transport through tailored geometries with SO interaction [80, 81, 82], magnetic fields [83, 84, 85] or a combination of both [86] can result in a significant spin filter effect.

As a representative example for the methods mentioned above, in the present section we present spin filtering due to Rashba SO interaction in quantum wires connected to two terminals. We consider a quantum wire in $y$-direction realized in a 2DEG in the $(x, y)$ plane connected to two nonmagnetic leads. The Hamiltonian of the system, whit spatially dependent Rashba SO interaction is given by

$$
H_{0}=\frac{\hat{p}^{2}}{2 m^{*}}+\frac{\alpha(x)}{2 \hbar}\left(\hat{\sigma}_{x} \hat{p}_{y}-\hat{\sigma}_{y} \hat{p}_{x}\right)+\left(\hat{\sigma}_{x} \hat{p}_{y}-\hat{\sigma}_{y} \hat{p}_{x}\right) \frac{\alpha(x)}{2 \hbar}+V(x, y)+U_{\mathrm{B}}(x, y) .
$$

Here $V(x, y)$ is the lateral transverse confinement potential forming the quantum wire, while $U_{\mathrm{B}}(x, y)$ is an additional electrostatic potential in the system, e.g., realized by gate voltages. Furthermore, $\hat{\sigma}_{i}$ denote the Pauli spin matrices, and $m^{*}$ is the effective electron mass of the semiconducting material. We consider a constant Rashba SO interaction strength $\left(=\alpha_{\mathrm{C}}\right)$ in the central region of the system, which is connected to two semi-infinite leads on opposite sides, where $\alpha(x)$ is chosen to be zero avoiding ambiguities in the definition of spin current that arise for leads with SO interaction [87]. In order not to introduce additional effects due to an abrupt jump in the SO coupling strength, the parameter $\alpha(x)$ is changed sufficiently smooth from zero to $\alpha_{\mathrm{C}}$ between the leads and the central region. For 
the numerical calculations presented in the next two sections the Hamiltonian (24) is discretized as shown in Sec. III yielding a tight-binding Hamiltonian on a square grid.

In the rest of this section we investigate the transport properties in the linear response regime due to an infinitesimal bias voltage $\delta U$ applied between the left and right contact. The charge (C) and spin (S) current in the Landauer formalism are then given by

$$
I_{\mathrm{C} / \mathrm{S}}=G_{\mathrm{C} / \mathrm{S}} T_{\mathrm{C} / \mathrm{S}} \delta U
$$

where $G_{\mathrm{C}}=e^{2} / h$ and $G_{\mathrm{S}}=e / 4 \pi$ are the conductance quanta of charge and spin respectively. Since, opposite to charge current, the spin current can be different in the right and left lead [85], here we choose to evaluate the respective currents in the right lead. Then the transmission probabilities $T_{\mathrm{C} / \mathrm{S}}$ at the Fermi energy are given by

$$
T_{\mathrm{C}}=T_{+,+}+T_{+,-}+T_{-,+}+T_{-,-}, \quad T_{\mathrm{S}}=T_{+,+}+T_{+,-}-T_{-,+}-T_{-,-},
$$

where $T_{\sigma, \sigma^{\prime}}=\sum_{n \in \mathrm{R}, n^{\prime} \in \mathrm{L}}\left|S_{n, \sigma ; n^{\prime}, \sigma^{\prime}}\right|^{2}$ is the probability for an electron, injected into the left (L) lead with spin state $\sigma^{\prime}$ to be transmitted to the right (R) lead and end up there in spin state $\sigma$. In the present and the following section we fix the spin quantization axis to the $y$-axis. The scattering matrix elements $S_{n, \sigma ; n^{\prime}, \sigma^{\prime}}$ and therefore also the spin resolved transmission probabilities $T_{\sigma, \sigma^{\prime}}$ are evaluated using the recursive Greens function algorithm presented in Secs. III and IV]

One general feature of Landauer transport in a quantum wire with SO interaction and non-magnetic leads is the absence of spin-polarized currents in a lead that supports only a single transversal mode. This property can be derived from the invariance of the system under the time-reversal operator $\mathcal{T}=-i \hat{\mathcal{C}} \sigma_{y}$ [88], where $\hat{\mathcal{C}}$ is the operator of complex conjugation. For a perfect quantum wire which is translationally invariant in the direction of transport all occupied transversal subbands transmit without reflection and spin polarization is not possible due to SO interaction. However, if backreflection, caused by deviations from a perfect quantum wire, is present, it is possible to observe spin-polarized currents in leads with at least two transversal channels. There the typical mechanism responsible for spin polarization is the mixing of spins from different transversal subbands due to the SO interaction.

In Eq. (24) this translational invariance in $y$-direction is already broken by the spatially varying SO interaction $\alpha(x)$ even if the quantum wire was perfect in $y$-direction otherwise, i.e. $V(x, y)=V(y)$ and $U_{\mathrm{B}}(x, y)=U_{\mathrm{B}}(y)$. However, if the region where $\alpha(x)$ is turned on/off is sufficiently long, reflection due to the change of $\alpha(x)$ is negligible.

There exist several device proposals relying on this mixing of spins from different transversal subbands due to $x$ dependent lateral confinement potentials $V(x, y)$ or other superimposed electrostatic potentials $U_{\mathrm{B}}(x, y)$. These device designs include, e.g., constrictions [80, 89], lateral side pockets [81], or electrostatic barriers [82], to name only a few.

In most of those proposals, systems symmetric with respect to inversion of the $x$-coordinate were considered, i.e. $V(x, y)=V(-x, y), U_{\mathrm{B}}(x, y)=U_{\mathrm{B}}(-x, y)$ and $\alpha(x)=\alpha(-x)$. Then the Hamiltonian (24) is left invariant upon application of the symmetry operation

$$
\hat{\mathcal{P}}=-\mathrm{i} \hat{\mathcal{C}} \hat{R}_{x} \hat{\sigma}_{z}
$$

where the operator $\hat{R}_{x}$ inverses the $x$-coordinate. The scattering wavefunctions inside the leads are changed by the operator $\hat{\mathcal{P}}$ in the following way: $\hat{R}_{x}$ exchanges the leads, i.e., a transversal mode index $n$ is replaced by the corresponding mode index $\bar{n}$ in the other lead. The operator of complex conjugation transforms incoming (outgoing) states into outgoing (incoming) states with complex conjugated amplitude. Moreover, the combined effect of $\hat{\mathcal{C}} \sigma_{z}$ is to rotate a spinor with the coordinates $(\theta, \phi)$ on the Bloch sphere to the coordinates $(\theta,-\phi+\pi)$. Exploiting this invariance of the Hamiltonian one can derive the relation $S_{n, \sigma ; n^{\prime}, \sigma^{\prime}}(E)=S_{\bar{n}^{\prime}, \sigma^{\prime} ; \bar{n}, \sigma}(E)$ between the elements of the scattering matrix (see Refs. [85, 88] for related expressions). This results in the equality of the spin flip transmissions $T_{+,-}=T_{-,+}$. Therefore, for those devices to be able to work as a spin filter, in view of Eq. (25) the spin conserving transmissions $T_{+,+}$and $T_{-,-}$need to be different.

As an example, in the following we consider Landauer transport through a Rashba SO quantum wire with a constriction. Similar calculations were carried out in Refs. [71, 80] where it was shown, that this setup is able to produce a spin polarized current of sizeable quantity. It was conjectured, that the mechanism responsible for the spin polarization was the depletion of higher transversal modes of the wire and a subsequent spin dependent repopulation of those modes when traversing the constriction [80]. To experimentally observe the predicted spin polarization the use of a transverse electron focusing technique was suggested [71].

A typical grid (with lattice spacing $a$ ) used in the calculation for the symmetric point contact in a wire of width $W=15 a$ is shown in Fig. $12 \mathrm{k}$, where the constriction of length $L_{\mathrm{PC}}=10 a$ is formed by hard-wall potentials:

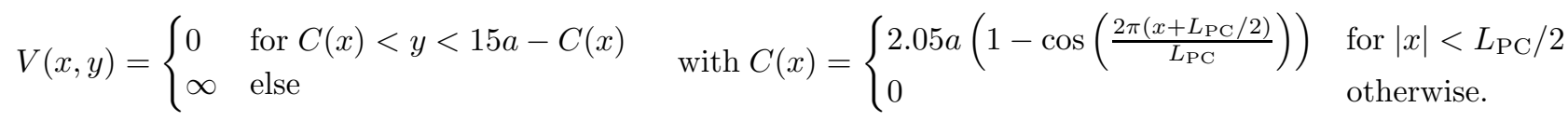




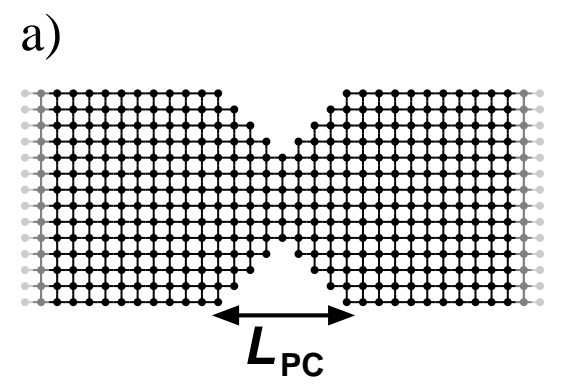

b)

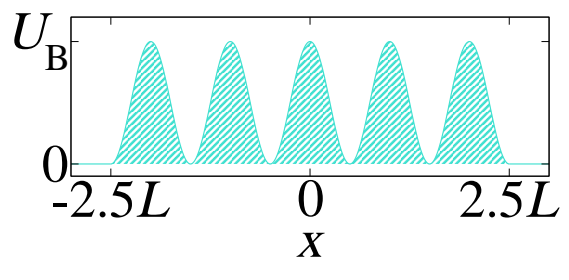

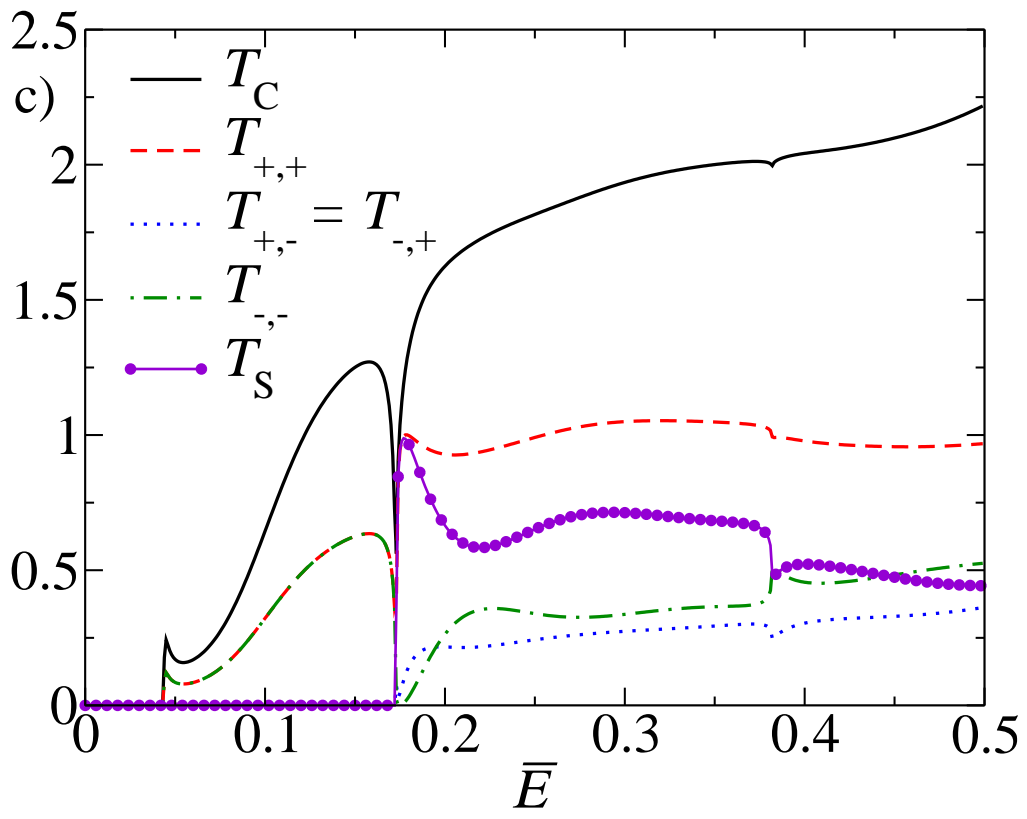

FIG. 12: Panel a) The square lattice discretization of a quantum wire (width $W=15 a$ ) with a single constriction of length $L_{\mathrm{PC}}=10 a$, see also Eq. (27). Panel b) Periodic array of $N=5$ electrostatic barriers with period length $L$ and barrier height $U_{\mathrm{B}}$. Panel c) Charge $T_{\mathrm{C}}$, spin $T_{\mathrm{S}}$ and spin resolved transmission probabilities $T_{\sigma, \sigma^{\prime}}$ for the system depicted in panel a) and specified in the text at fixed SO interaction strength $\bar{\alpha}=\frac{m^{*} a}{\hbar^{2}} \alpha_{\mathrm{C}}=0.1$ with respect to the Fermi energy $\bar{E}=\frac{2 m^{*} a^{2}}{\hbar^{2}} E$. The $n$-th transversal mode in the wire opens at $\bar{E}_{n}=\frac{\pi^{2} n^{2}}{(W / a)^{2}}$.

Additionally $U_{\mathrm{B}}(x, y)$ is set to zero. In Fig. 12k we present the relevant transmission probabilities with respect to the Fermi energy $E$ for this system. There we observe that the total conductance $T_{\mathrm{C}}$ is reduced in comparison with that of a perfect quantum wire. In the latter case $T_{\mathrm{C}}$ exhibits sharp steps due to conductance quantization [90, 91] which are washed out here due to tunneling processes through the constriction. Furthermore, for energies, where only a single transversal mode is supported in the quantum wire, the spin transmission vanishes as expected, i.e. $T_{+,+}=T_{-,-}$for energies $\bar{E} \lesssim 0.175$. Also the relation $T_{+,-}=T_{-,+}$is fulfilled as required by the symmetry of the setup. Finally, at energies where a new transversal mode opens up $\left(\bar{E}_{2} \approx 0.18, \bar{E}_{3} \approx 0.39\right)$ dips in the transmission probabilities become apparent. Those dips can be explained by interference of localized states in the central region where $\alpha(x)=\alpha_{\mathrm{C}}$ and the scattering states in the quantum wire where $\alpha(x)=0$ [92].

In order to study the influence of the adiabaticity of the constriction on the degree of spin polarization that can be extracted, in Fig. 13 we show $T_{\mathrm{S}}$ as a function of the length of the constriction $L_{\mathrm{PC}}$ for several values of $\alpha_{\mathrm{C}}$. For all of the curves we clearly observe an increase in spin transmission with increasing $L_{\mathrm{PC}}$, in accordance with the mechanism suggested in Ref. [80]. There it was argued in the context of Landau-Zener transitions that the repopulation of higher transversal subbands will be more efficient for more adiabatic constrictions or barriers, resulting in a higher degree of spin polarization. For $\bar{\alpha}=\frac{m^{*} a}{\hbar^{2}} \alpha_{\mathrm{C}}=0.1$ the spin transmission even approaches the maximal possible value $T_{\mathrm{S}}=2$. One drawback of the presented system is its restriction to unidirectional spin polarization. In agreement with the model of Ref. [80], Figs. 12 and 13 give evidence that $T_{\mathrm{S}} \geq 0$ for the parameter range considered. This limitation to output current with fixed spin polarization direction restricts the usability of the spin filter to special purposes. A possible way to circumvent this constraint is the use of a periodic array of electrostatic barriers [82], which we now briefly investigate. Fig. 14 shows the spin transmission of a straight hard-wall quantum wire $(C(x)=0$ in Eq. (27)) subject to $N=5$ electrostatic barriers,

$$
U_{\mathrm{B}}(x, y)=U_{\mathrm{B}}(x)= \begin{cases}U_{\mathrm{Barr}}\left(0.5-0.5 \cos \left(\frac{2 \pi(x+L / 2)}{L}\right)\right) & \text { for }-N L / 2<x<N L / 2 \\ 0 & \text { otherwise }\end{cases}
$$

(as shown in Fig. 12b). The spin transmission is plotted as a function of the Fermi energy and the SO interaction strength. Again, one observes $T_{\mathrm{S}}=0$ for energies below $\bar{E}_{2}$. Furthermore, different regions in parameter space exhibit opposite sign of $T_{\mathrm{S}}$, enabling to change the sign of the output polarization, e.g., by tuning $\alpha_{c}$ via gate voltages [25]. This additional functionality is due to resonant tunneling, which is absent for a quantum wire with only a single 


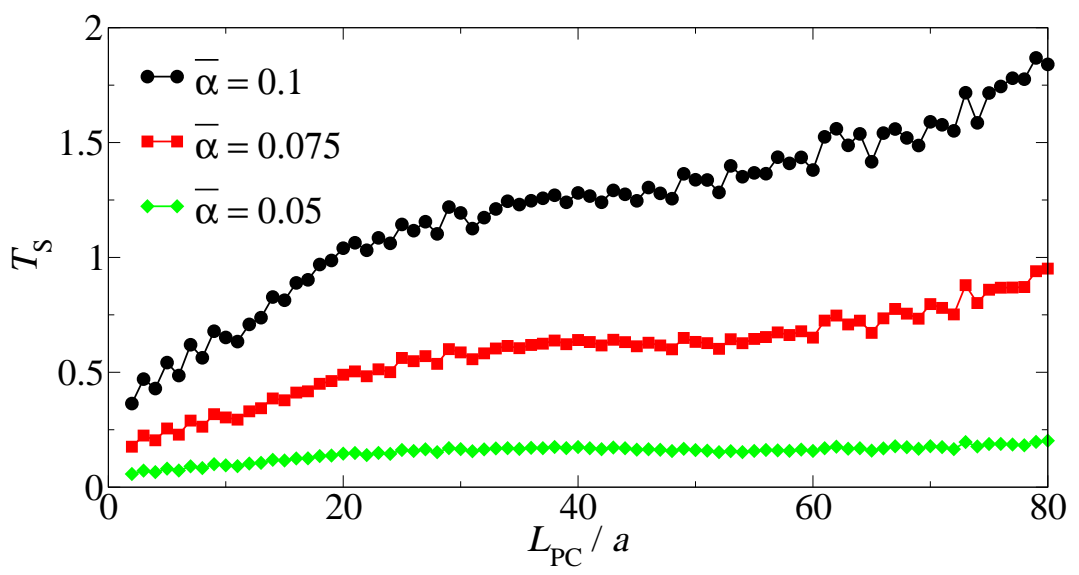

FIG. 13: Spin transmission probability $T_{\mathrm{S}}$ at fixed injection energy $\bar{E}=0.25$ within the second transversal mode for three different SO interaction strengths $\bar{\alpha}=0.1$ (black dots), $\bar{\alpha}=0.075$ (red squares) and $\bar{\alpha}=0.05$ (green diamonds) with respect to the length of the constriction $L_{\mathrm{PC}}$.

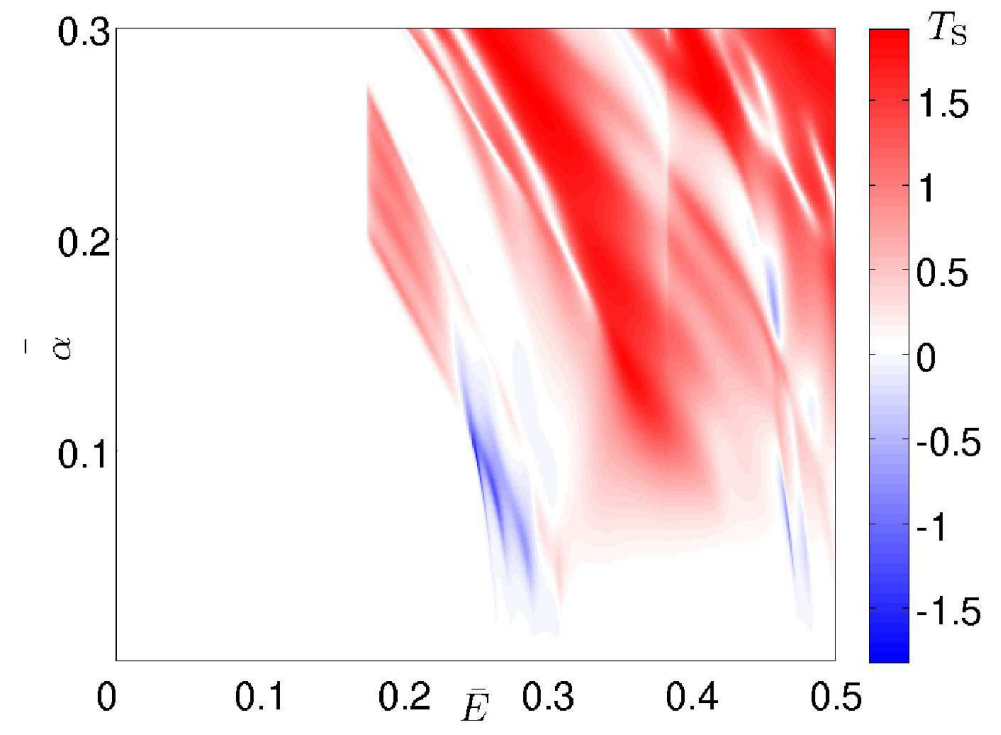

FIG. 14: Spin transmission probability $T_{\mathrm{S}}$ plotted as a function of the Fermi energy $\bar{E}$ and SO interaction strength $\bar{\alpha}$ for a quantum wire with $N=5$ electrostatic barriers of length $L=10 a$ and height $\bar{U}_{\mathrm{Barr}}=\frac{2 m^{*} a^{2}}{\hbar^{2}} U_{\mathrm{Barr}}=0.2$.

constriction or barrier.

\section{PURE SPIN CURRENT GENERATION}

In the preceding sections we focused on mesoscopic geometries that exhibit functionalities such as spin filtering or spin switching when applying an external bias between the contacts in the system. In the case of a static dc bias in a two terminal geometry those spin currents arise due to different currents of spin-up and spin-down electrons flowing into the direction of the contact with the lower chemical potential. However, over the last few years a growing number of device proposals has been put forward, that exhibit the interesting feature of pure spin current generation, i.e. spin currents in the absence of net charge transport. This intriguing case appears, when the direction of motion of spin-up electrons is opposite to the direction of motion of spin-down electrons and both currents equal in absolute magnitude.

Among the devices sharing the prospect of creating pure mesoscopic spin currents are systems with more than 
two terminals realized in 2DEGs exhibiting Rashba [2, 3] and/or the Dresselhaus [93] SO coupling. Here a charge current is induced in this multiterminal structure by the application of bias voltages between the different contacts of the system. However, if the voltage of one of the leads is adjusted to make it work as a voltage probe, no charge current passes this lead but a pure spin current can appear owing to the SO coupling present in the system. The basic working principle behind these devices is the so-called mesoscopic spin Hall effect [94], a version of the intrinsic spin Hall effect [27], where the typical system size does not exceed the phase coherence length of the electrons. The SO interaction leads to different transport dynamics for different spin species, which can be used to extract the desired pure spin currents by a clever design of the multiterminal geometry [21, 95, 96].

Complementary to the generation of pure spin current in multiterminal geometries, there are other types of devices not relying on the application of a net dc-bias. In spin pumping, the cyclic variation of two or more system parameters, such as e.g. gate voltages, induces spin-polarized currents at zero bias, where the induced charge current can be tuned to disappear, leaving pure spin currents. Several realizations of spin pumps in mesoscopic systems have been proposed, relying on SO interaction [97, 98] or the Zeeman coupling of electrons to external magnetic fields [99]. The latter proposal has been experimentally confirmed [100] by detecting spin-polarized currents making use of a transverse electron focusing technique [3] mentioned in the previous section.

Complementary to pumps, ratchets only require a single driving parameter to achieve directed transport, and the current direction can be switched upon tuning external parameters such as temperature. In addition to the requirement of a broken spatial symmetry the ratchet has to be operated out of thermal equilibrium. The concept of particle ratchets, which has been addressed in numerous works [101], has recently been extended to systems called spin ratchets. To be specific, the mesoscopic spin ratchets proposed so far [82, 85, 102], are based on a quantum wire realized in a 2DEG. Between the two contacts attached to the quantum wire an ac bias voltage $U_{\mathrm{R}}(t)$ is applied (rocking ratchet) with zero net (time-averaged) bias. Furthermore, in the central region of the quantum wire the spin degeneracy is lifted due to either SO interaction [82] or the Zeeman coupling to an external magnetic field [85, 102]. Upon appropriate choice of the system geometry and tuning of the external driving the charge transported in the forward $\left(U_{\mathrm{R}}>0\right)$ and backward bias $\left(U_{\mathrm{R}}<0\right)$ situation can be made equal allowing for spin currents in the absence of net charge transport.

In the following we outline the model for the spin ratchets introduced in Refs. [82, 85, 102]. There, driving with a period $t_{0}$ is considered. It is implied, that this period is much larger than characteristic time scales related to the electron transport through the quantum wire. Therefore, the system is assumed to be in a steady state at every instance of time, and the Landauer-Büttiker approach to transport is used for the computation of the ratchet currents. To be specific, we consider an unbiased square wave driving $U_{\mathrm{R}}(t)=U_{0} \operatorname{sign}\left[\sin \left(2 \pi t / t_{0}\right)\right]$, where $U_{\mathrm{R}}(t)$ is restricted to the values $\pm U_{0}$. The net current is then given by the average of the steady-state currents in the two rocking situations (labeled $+U_{0}$ and $-U_{0}$ respectively) for both charge and spin,

$$
\left\langle I_{\mathrm{C} / \mathrm{S}}\left(U_{0}\right)\right\rangle=\left[I_{\mathrm{C} / \mathrm{S}}\left(+U_{0}\right)+I_{\mathrm{C} / \mathrm{S}}\left(-U_{0}\right)\right] / 2 .
$$

Since the spin ratchet effect requires nonlinear transport [102], i.e. finite bias voltages, the Hamiltonian (24) introduced in the previous section has to be extended to additionally include the effective electrostatic potential in the conductor due to the applied bias. Therefore, we add the term $H_{\mathrm{R}}=e U_{\mathrm{R}} g\left(x, y ; U_{\mathrm{R}}\right)$ to the Hamiltonian (24) yielding the full Hamiltonian at finite bias:

$$
H=H_{0}+H_{\mathrm{R}}
$$

Furthermore at finite bias a generalized version of the expressions for charge and spin current valid at $U_{\mathrm{R}} \neq 0$ has to be used. For coherent Landauer transport those currents can be obtained from an integration of the transmission probabilities over the Fermi window [1]. Finally, the averaged charge $\left\langle I_{\mathrm{C}}\right\rangle$ and spin $\left\langle I_{\mathrm{S}}\right\rangle$ currents can be written as [85]

$$
\left\langle I_{\mathrm{C} / \mathrm{S}}\left(U_{0}\right)\right\rangle=\frac{G_{\mathrm{C} / \mathrm{S}}}{2 e} \int_{E_{\mathrm{C}}}^{\infty} \mathrm{d} E \Delta f\left(E, U_{0}\right) \Delta T_{\mathrm{C} / \mathrm{S}}\left(E, U_{0}\right) .
$$

Here $E_{\mathrm{C}}$ is the energy of the conduction band edge and $\Delta f\left(E, U_{0}\right)=\left[f\left(E, E_{\mathrm{F}}+e U_{0} / 2\right)-f\left(E, E_{\mathrm{F}}-e U_{0} / 2\right)\right]$ is the difference between the Fermi functions of the leads at bias voltage $U_{0}$, defining the Fermi window. The averaged charge/spin transmission is just the difference between the steady state transmissions in the two rocking situations:

$$
\Delta T_{\mathrm{C} / \mathrm{S}}\left(E, U_{0}\right)=T_{\mathrm{C} / \mathrm{S}}\left(E,+U_{0}\right)-T_{\mathrm{C} / \mathrm{S}}\left(E,-U_{0}\right) .
$$

Considering the Hamiltonian (29) we now show under what conditions the net charge transported after one full rocking period is zero, i.e., $\left\langle I_{\mathrm{C}}\left(U_{0}\right)\right\rangle=0$. If the electrostatic potentials $V(x, y), U_{\mathrm{B}}(x, y)$ and the Rashba SO strength $\alpha(x)$ are invariant under inversion of the $x$-coordinate,

$$
V(x, y)=V(-x, y), \quad U_{\mathrm{B}}(x, y)=U_{\mathrm{B}}(-x, y), \quad \alpha(x)=\alpha(-x),
$$




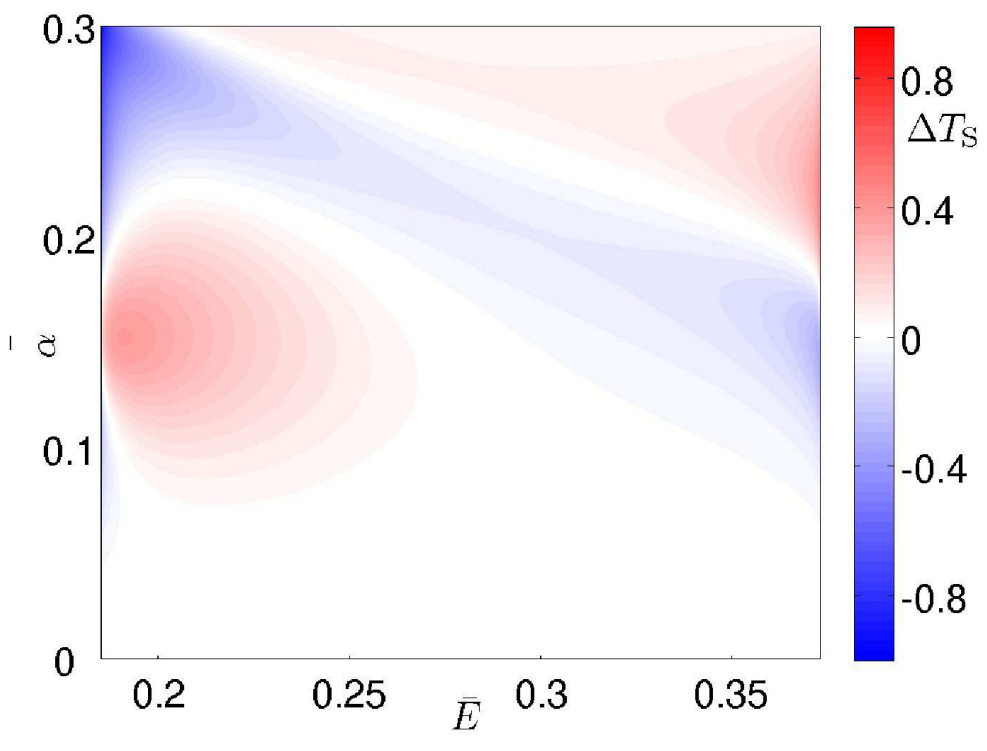

FIG. 15: Net ratchet spin transmission probability $\Delta T_{\mathrm{S}}\left(U_{0}\right)=2\left(T_{+,-}\left(+U_{0}\right)-T_{-,+}\left(+U_{0}\right)\right)$ for a stripe with a junction (see text) presented as a function of injection energy $\bar{E}$ and SO interaction strength $\bar{\alpha}$ at finite applied voltage $e U_{0}=0.02 \frac{\hbar^{2}}{2 m^{*} a^{2}}$. Note the sign change in the spin transmission upon tuning the SO coupling.

it is appropriate to assume that the electrostatic potential distribution due to the finite applied voltage $g\left(x, y ; U_{\mathrm{R}}\right)$ also possesses this symmetry. Then the total Hamiltonian (29) is invariant under the action of the symmetry operation $\hat{\mathcal{P}}=-\mathrm{i} \hat{\mathcal{C}} \hat{R}_{\mathrm{U}} \hat{R}_{x} \sigma_{z}$ where $R_{\mathrm{U}}$ switches the sign of the bias voltage $\left( \pm U_{0} \leftrightarrow \mp U_{0}\right)$, yielding the relation

$$
T_{\sigma, \sigma^{\prime}}\left(E, \pm U_{0}\right)=T_{\sigma^{\prime}, \sigma}\left(E, \mp U_{0}\right)
$$

between the spin-resolved transmission probabilities in the two rocking situations [85]. Inserting this relation into Eq. (30), we observe that the expression for the net charge transmission $\Delta T_{\mathrm{C}}$ is zero, resulting in vanishing net charge current. Furthermore it can be used to simplify the expression for the net spin current:

$$
\left\langle I_{\mathrm{S}}\left(U_{0}\right)\right\rangle=\frac{G_{\mathrm{S}}}{e} \int_{E_{\mathrm{C}}}^{\infty} \mathrm{d} E \Delta f\left(E ; U_{0}\right)\left[T_{+,-}\left(E,+U_{0}\right)-T_{-,+}\left(E,+U_{0}\right)\right] .
$$

At $U_{0}=0$ the Hamiltonian (29) reduces to Eq. (24), which is invariant under the operation of Eq. (26), yielding $T_{+,-}(E, 0)=T_{-,+}(E, 0)$. This absence of net spin current $\left\langle I_{\mathrm{S}}\left(U_{0}=0\right)\right\rangle=0$ in the linear response regime is in agreement with the theoretical prediction [85]. However, for finite rocking voltages $U_{0} \neq 0$ the additional potential introduced via $H_{\mathrm{R}}$ breaks this symmetry and therefore enables different spin flip transmissions and thus a resulting net spin current.

We now turn our attention to the quantum wire shown in Fig. 12k. For this system, which exhibits the symmetries of Eq. (31), we perform numerical calculations at finite bias $U_{0}$, in order to confirm its operability as a spin ratchet. In general, the function $g\left(x, y ; U_{\mathrm{R}}\right)$ has to be obtained by self-consistently solving the Schrödinger and Poisson equation of the system. However, in the present treatment we make use of a simple model for $g\left(x, y ; U_{\mathrm{R}}\right)$ assuming a linear voltage drop in the region where the point contact is formed in the quantum wire:

$$
g\left(x, y ; U_{\mathrm{R}}\right)=g(x)= \begin{cases}1 / 2 & \text { for } x<-L_{\mathrm{PC}} / 2 \\ -x / L_{\mathrm{PC}} & \text { for }-L_{\mathrm{PC}} / 2<x<L_{\mathrm{PC}} / 2 \\ -1 / 2 & \text { for } x>L_{\mathrm{PC}} / 2\end{cases}
$$

It is well known that a constriction in a quantum wire acts as an effective potential barrier constituting a region where the voltage applied across the wire is likely to drop. Since the bias voltages we consider are small compared to the energy shift introduced by the constriction, this assumption of a linear voltage drop should be an appropriate approximation for the actual distribution of the electrostatic potential in this wire [103]. 
In Fig. 15 we plot the net spin transmission $\Delta T_{\mathrm{S}}$ as a function of $\bar{\alpha}$ and the injection energy in the range, where both leads support two transversal modes. We observe that $\left|\Delta T_{\mathrm{S}}\right|$ reaches values of up to 0.9 in the parameter range shown. Furthermore, for a given value of injection energy, the sign of $\Delta T_{\mathrm{S}}$ can be switched by changing $\alpha_{\mathrm{C}}$. Since the strength of the Rashba SO interaction $\alpha_{\mathrm{C}}$ can be tuned via gate voltages [25] the presented system offers also the possibility for experimentally steering and switching the spin current direction.

\section{FUTURE DIRECTIONS}

In the present work we outlined general theoretical and computational concepts of coherent spin-dependent transport at low temperatures and focussed, with regard to numerical examples and possible experimental realizations, onto ballistic two-dimensional nanostructures based on non-magnetic high-mobility semiconductors.

In order to experimentally achieve high spin polarizations and reasonable spin currents, if possible at room temperature, broad efforts are made to investigate and design novel materials for spintronics. Here, prominent and promising examples, both with respect to fundamental physics and possible applications, are magnetic semiconductors such as GaMnAs 104] or semimagnetic materials with huge $g$-factors, for instance HgTe 105]. Charge transport in these materials is based on holes. However, relatively few theoretical papers deal with phase coherence effects for hole (spin) transport, though the rich band structure and the interplay between heavy and light hole (or electron- and hole-like) degree of freedoms promise interesting additional features.

The theoretical methods for quantum transport, presented here in the context of mesoscopic systems, are also applied and extended to treat transport in a further prospective field, namely through single-molecule junctions [106], for instance (break) junctions with a molecule bridging the gap between two leads or scanning tunneling microscope measurements of tunnel current through molecules at surfaces. In Molecular Spintronics [44, 107] spin effects in transport through molecules are addressed. This subfield of spin electronics is still in its infancy. On the computational side these systems pose considerable problems since an adequate approach requires an appropriate description of the electronic and possibly vibrational properties of the molecule including the coupling to and effects of the leads. Whether (spin) DFT calculations for such an embedded molecule, combined with Landauer-type transport calculations, are appropriate, remains to be an issue, in particular if charging or non-equilibrium effects are involved.

As a further future direction we expect that spin transport in graphene, monolayers of graphite, may evolve as another future research line. After its experimental discovery in 2004 [108], graphene has gained much experimental and huge theoretical attention owing to its many exotic properties such as the massless charge carriers, internal spin-like degree of freedoms and unconventional transport characteristics. Also first experiments on graphene-based nanoconductors, e.g., measurements of the Aharonov-Bohm effect in graphene rings [109], are on their way. Graphene is also viewed as a prospective candidate for spin-electronics, since the spin decoherence and spin relaxation times in graphene are expected to be long [110, 111]. Recent promising experiments already succeeded in injecting spins from ferromagnetic metallic contacts into graphene, although the conductance mismatch between graphene and the ferromagnetic leads is expected to suppress the efficiency. Recent theoretical proposals predict efficient spin injection into bulk graphene from graphene ribbons employing the occurrence of current-carrying spin-polarized edge states in the ribbons [112].

\section{ACKNOWLEDGEMENTS}

Parts of the results presented are based on previous work together with D. Bercioux, D. Frustaglia, and A. Pfund. We further thank I. Adagideli, M. Grifoni and D. Weiss for useful discussions. Two of us, KR and MW, acknowledge financial support by the Deutsche Forschungsgemeinschaft (SFB 689) and MS through the Studienstiftung des Deutschen Volkes.

[1] Datta S (2002) Electronic Transport in Mesoscopic Systems. Cambridge University Press, Cambridge.

[2] Rashba EI (1960) Properties of semiconductors with an extremum loop .1. cyclotron and combinational resonance in a magnetic field perpendicular to the plane of the loop. Sov Phys Solid State 2:1109-1122.

[3] Bychkov YA, Rashba EI (1984) Oscillatory effects and the magnetic susceptibility of carriers in inversion layers. J Phys C: Solid State Phys 17:6039-6045.

[4] Frustaglia D, Richter K (2004) Spin interference effects in ring conductors subject to Rashba coupling. Phys Rev B 69:235310. 
[5] Hentschel M, Schomerus H, Frustaglia D, Richter K (2004) Aharonov-Bohm physics with spin. I. Geometric phases in one-dimensional ballistic rings. Phys Rev B 69:155326.

[6] Cohen G, Hod O, Rabani E (2007) Constructing spin interference devices from nanometric rings. arXiv:0710.4770v1.

[7] Loss D, Goldbart P, Balatsky AV (1990) Berry's phase and persistent charge and spin currents in textured mesoscopic rings. Phys Rev Lett 65:1655-1658.

[8] Stern A (1992) Berry's phase, motive forces, and mesoscopic conductivity. Phys Rev Lett 68:1022-1025.

[9] Aronov AG, Lyanda-Geller YB (1993) Spin-orbit Berry phase in conducting rings. Phys Rev Lett 70:343-346.

[10] Qian TZ, Su ZB (1994) Spin-orbit interaction and Aharonov-Anandan phase in mesoscopic rings. Phys Rev Lett 72:23112315.

[11] Frustaglia D, Hentschel M, Richter K (2001) Quantum transport in nonuniform magnetic fields: Aharonov-Bohm ring as a spin switch. Phys Rev Lett 87:256602.

[12] Frustaglia D, Hentschel M, Richter K (2004) Aharonov-Bohm physics with spin. II. Spin-flip effects in two-dimensional ballistic systems. Phys Rev B 69:155327.

[13] Nitta J, Bergsten T (2007) Time reversal Aharonov-Casher effect using Rashba spin-orbit interaction. New J Phys 9:341.

[14] Morpurgo AF, Heida JP, Klapwijk TM, van Wees BJ, Borghs G (1998) Ensemble-average spectrum of Aharonov-Bohm conductance oscillations: Evidence for spin-orbit-induced Berry's phase. Phys Rev Lett 80:1050-1053.

[15] Yau JB, De Poortere EP, Shayegan M (2002) Aharonov-Bohm oscillations with spin: Evidence for Berry's phase. Phys Rev Lett 88:146801.

[16] König M, Tschetschetkin A, Hankiewicz EM, Sinova J, Hock V, Daumer V, Schäfer M, Becker CR, Buhmann H, Molenkamp LW (2006) Direct observation of the Aharonov-Casher phase. Phys Rev Lett 96:076804.

[17] Grbić B, Leturcq R, Ihn T, Ensslin K, Reuter D, Wieck AD (2007) Aharonov-Bohm oscillations in the presence of strong spin-orbit interactions. Phys Rev Lett 99:176803.

[18] Nitta J, Meijer FE, Takayanagi H (1999) Spin-interference device. Appl Phys Lett 75:695-697.

[19] Mal'shukov AG, Shlyapin VV, Chao KA (1999) Effect of the spin-orbit geometric phase on the spectrum of AharonovBohm oscillations in a semiconductor mesoscopic ring. Phys Rev B 60:R2161-R2164.

[20] Splettstoesser J, Governale M, Zülicke U (2003) Persistent current in ballistic mesoscopic rings with Rashba spin-orbit coupling. Phys Rev B 68:165341.

[21] Souma S, Nikolić BK (2005) Spin Hall current driven by quantum interferences in mesoscopic Rashba rings. Phys Rev Lett 94:106602.

[22] Popp M, Frustaglia D, Richter K (2003) Spin filter effects in mesoscopic ring structures. Nanotechnology 14:347-351.

[23] Bellucci S, Onorato P (2007) Filtering of spin currents based on a ballistic ring. J Phys: Condens Matter 19:395020.

[24] Ionicioiu R, D’Amico I (2003) Mesoscopic Stern-Gerlach device to polarize spin currents. Phys Rev B 67:041307.

[25] Nitta J, Akazaki T, Takayanagi H, Enoki T (1997) Gate control of spin-orbit interaction in an inverted $\mathrm{In}_{0.53} \mathrm{Ga}_{0.47} \mathrm{As} / \mathrm{In}_{0.52} \mathrm{Al}_{0.48}$ As heterostructure. Phys Rev Lett 78:1335-1338.

[26] Schmidt G, Ferrand D, Molenkamp LW, Filip AT, van Wees BJ (2000) Fundamental obstacle for electrical spin injection from a ferromagnetic metal into a diffusive semiconductor. Phys Rev B 62:R4790-R4793.

[27] Sinova J, Culcer D, Niu Q, Sinitsyn NA, Jungwirth T, MacDonald AH (2004) Universal intrinsic spin Hall effects. Phys Rev Lett 92:126603.

[28] Focus on Spintronics in Reduced Dimensions, New J. Phys. 9 (2007). Test.

[29] Corresponding settings, where a precessing magnetization in a ferromagnet emits spin currents, were reviewed by Tserkovnyak Y, Brataas A, Bauer GEW, Halperin BI (2005) Nonlocal magnetization dynamics in ferromagnetic heterostructures. Rev Mod Phys 77:1375.

[30] Landauer R (1957) Spatial variation of currents and fields due to localized scatterers in metallic conduction. IBM J Res Dev 1:223-231.

[31] Büttiker M, Imry Y, Landauer R, Pinhas S (1985) Generalized many-channel conductance formula with application to small rings. Phys Rev B 31:6207-6215.

[32] Stone AD, Szafer A (1988) What is measured when you measure a resistance? - The Landauer forumula revisited. IBM J Res Dev 32:384-413.

[33] Ferry DK, Goodnick SM (2001) Transport in Nanostructures. Cambridge University Press, Cambridge.

[34] Fisher DS, Lee PA (1981) Relation between conductivity and transmission matrix. Phys Rev B 23:6851-6854.

[35] Baranger HU, Stone AD (1989) Electrical linear-response theory in an arbitrary magnetic field: A new fermi-surface formation. Phys Rev B 40:8169-8193.

[36] Kimball GE, Shortley GH (1934) The numerical solution of Schrödinger's equation. Phys Rev 45:815-820.

[37] Pauling L, Wilson EB (1935) Introduction to Quantum Mechanics. Dover, New York.

[38] Havu P, Havu V, Puska MJ, Nieminen RM (2004) Nonequilibrium electron transport in two-dimensional nanostructures modeled using Green's functions and the finite-element method. Phys Rev B 69:115325.

[39] Bowen RC, Klimeck G, Lake RK, Frensley WR, Moise T (1997) Quantitative simulation of a resonant tunneling diode. J Appl Phys 81:3207-3213.

[40] Sanvito S, Lambert CJ, Jefferson JH, Bratkovsky AM (1999) General Green's-function formalism for transport calculations with spd Hamiltonians and giant magnetoresistance in Co- and Ni-based magnetic multilayers. Phys Rev B 59:1193611948.

[41] Luisier M, Schenk A, Fichtner W, Klimeck G (2006) Atomistic simulation of nanowires in the $s p^{3} d^{5} s^{*}$ tight-binding formalism: From boundary conditions to strain calculations. Phys Rev B 74:205323.

[42] Brandbyge M, Mozos JL, Ordejón P, Taylor J, Stokbro K (2002) Density-functional method for nonequilibrium electron 
transport. Phys Rev B 65:165401.

[43] Di Carlo A, Pecchia A, Latessa L, Frauenheim T, Seifert G (2006) Tight-binding DFT for molecular electronics (gDFTB). In: Cuniberti G, Fagas G, Richter K (eds) Introducing Molecular Electronics, Springer, Berlin, Heidelberg, pp 153-184.

[44] Rocha AR, García-Suárez VM, Bailey S, Lambert C, Ferrer J, Sanvito S (2006) Spin and molecular electronics in atomically generated orbital landscapes. Phys Rev B 73:085414.

[45] Lopez Sancho MP, Lopez Sancho JM, Rubio J (1984) Quick iterative scheme for the calculation of transfer matrices: application to Mo (100). J Phys F: Met Phys 14:1205-1215.

[46] Lopez Sancho MP, Lopez Sancho JM, Rubio J (1985) Highly convergent schemes for the calculation of bulk and surface Green functions. J Phys F: Met Phys 15:851-858.

[47] Krstić PS, Zhang XG, Butler WH (2002) Generalized conductance formula for the multiband tight-binding model. Phys Rev B 66:205319.

[48] Usuki T, Takatsu M, Kiehl RA, Yokoyama N (1994) Numerical analysis of electron-wave detection by a wedge-shaped point contact. Phys Rev B 50:7615-7625.

[49] Usuki T, Saito M, Takatsu M, Kiehl RA, Yokoyama N (1995) Numerical analysis of ballistic-electron transport in magnetic fields by using a quantum point contact and a quantum wire. Phys Rev B 52:8244-8255.

[50] Lambert CJ, Weaire D (1980) Decimation and Anderson localization. Phys Status Solid B 101:591-595.

[51] Leadbeater M, Lambert CJ (1998) A decimation method for studying transport properties of disordered systems. Ann Phys 7:498-502.

[52] Mamaluy D, Vasileska D, Sabathil M, Zibold T, Vogl P (2005) Contact block reduction method for ballistic transport and carrier densities of open nanostructures. Phys Rev B 71:245321.

[53] Thouless DJ, Kirkpatrick S (1981) Conductivity of the disordered linear chain. J Phys C: Solid State Phys 14:235-245.

[54] Lee PA, Fisher DS (1981) Anderson localization in two dimensions. Phys Rev Lett 47:882-885.

[55] MacKinnon A (1985) The calculation of transport properties and density of states of disordered solids. Z Phys B 59:385390.

[56] Baranger HU, DiVincenzo DP, Jalabert RA, Stone AD (1991) Classical and quantum ballistic-transport anomalies in microjunctions. Phys Rev B 44:10637-10675.

[57] Lake R, Klimeck G, Bowen RC, Jovanovic D (1997) Single and multiband modeling of quantum electron transport through layered semiconductor devices. J Appl Phys 81:7845-7869.

[58] Lassl A, Schlagheck P, Richter K (2007) Effects of short-range interactions on transport through quantum point contacts: A numerical approach. Phys Rev B 75:045346.

[59] Drouvelis P, Schmelcher P, Bastian P (2006) Parallel implementation of the recursive Green's function method. J Comp Phys 215:741-756.

[60] Rotter S, Tang JZ, Wirtz L, Trost J, Burgdörfer J (2000) Modular recursive Green's function method for ballistic quantum transport. Phys Rev B 62:1950-1960.

[61] Rotter S, Weingartner B, Rohringer N, Burgdörfer J (2003) Ballistic quantum transport at high energies and high magnetic fields. Phys Rev B 68:165302.

[62] Cuthill E, McKee J (1969) Reducing the bandwidth of sparse symmetric matrices. In: Proc. ACM National Conference. Association for Computing Machinery, New York, pp 157-172.

[63] George A (1971) Computer implementation of the finite element method. Tech Rep STAN-CS-71-208, Computer Sci. Dept., Stanford Univ., Stanford, Calif.

[64] Liu WH, Sherman AH (1976) Comparative analysis of the Cuthill-McKee and the reverse Cuthill-McKee ordering algorithms for sparse matrices. SIAM J Num Anal 13:198-213.

[65] Gibbs NE, William G Poole J, Stockmeyer PK (1976) An algorithm for reducing the bandwidth and profile of a sparse matrix. SIAM J Num Anal 13:236-250.

[66] Wimmer M, Richter K (2007) A block-tridiagonalization algorithm for quantum transport. Unpublished.

[67] Whaley RC, Petitet A, Dongarra JJ (2001) Automated empirical optimization of software and the ATLAS project. Parallel Comput 27:3-35.

[68] van Houten H, Beenakker CWJ, Williamson JG, Broekaart MEI, van Loosdrecht PHM, van Wees BJ, Mooij JE, Foxon CT, Harris JJ (1989) Coherent electron focusing with quantum point contacts in a two-dimensional electron gas. Phys Rev B 39:8556-8575.

[69] Usaj G, Balseiro CA (2004) Transverse electron focusing in systems with spin-orbit coupling. Phys Rev B 70:041301.

[70] Govorov AO, Kalameitsev AV, Dulka JP (2004) Spin-dependent transport of electrons in the presence of a smooth lateral potential and spin-orbit interaction. Phys Rev B 70:245310.

[71] Reynoso A, Usaj G, Balseiro CA (2007) Detection of spin polarized currents in quantum point contacts via transverse electron focusing. Phys Rev B 75:085321.

[72] Rokhinson LP, Larkina V, Lyanda-Geller YB, Pfeiffer LN, West KW (2004) Spin separation in cyclotron motion. Phys Rev Lett 93:146601.

[73] Potok RM, Folk JA, Marcus CM, Umansky V (2002) Detecting spin-polarized currents in ballistic nanostructures. Phys Rev Lett 89:266602.

[74] Folk JA, Potok RM, Marcus CM, Umansky V (2003) A Gate-Controlled Bidirectional Spin Filter Using Quantum Coherence. Science 299:679-682.

[75] Rokhinson LP, Pfeiffer LN, West KW (2006) Spontaneous spin polarization in quantum point contacts. Phys Rev Lett 96:156602.

[76] Fabian J, Das Sarma S (2002) Spin transport in inhomogeneous magnetic fields: A proposal for Stern-Gerlach-like 
experiments with conduction electrons. Phys Rev B 66:024436.

[77] Kiselev AA, Kim KW (2001) T-shaped ballistic spin filter. Appl Phys Lett 78:775-777.

[78] Ohe JI, Yamamoto M, Ohtsuki T, Nitta J (2005) Mesoscopic stern-gerlach spin filter by nonuniform spin-orbit interaction. Phys Rev B 72:041308.

[79] Cummings AW, Akis R, Ferry DK (2006) Electron spin filter based on rashba spin-orbit coupling. Appl Phys Lett 89:172115.

[80] Eto M, Hayashi T, Kurotani Y (2005) Spin polarization at semiconductor point contacts in absence of magnetic field. J Phys Soc Jpn 74:1934.

[81] Zhai F, Xu HQ (2007) Spin filtering and spin accumulation in an electron stub waveguide with spin-orbit interaction. Phys Rev B 76:035306.

[82] Scheid M, Pfund A, Bercioux D, Richter K (2007) Coherent spin ratchets: A spin-orbit based quantum ratchet mechanism for spin-polarized currents in ballistic conductors. Phys Rev B 76:195303.

[83] Song JF, Ochiai Y, Bird JP (2003) Fano resonances in open quantum dots and their application as spin filters. Appl Phys Lett 82:4561-4563.

[84] Zhai F, Xu HQ (2006) Spin filtering in single magnetic barrier structures revisited. Appl Phys Lett 88:032502.

[85] Scheid M, Bercioux D, Richter K (2007) Zeeman ratchets: pure spin current generation in mesoscopic conductors with non-uniform magnetic fields. New J Phys 9:401.

[86] Zhai F, Xu HQ (2005) Generation of spin polarization in two-terminal electron waveguides by spin-orbit interaction and magnetic field modulations. Phys Rev B 72:085314.

[87] Shi J, Zhang P, Xiao D, Niu Q (2006) Proper definition of spin current in spin-orbit coupled systems. Phys Rev Lett 96:076604.

[88] Zhai F, Xu HQ (2005) Symmetry of spin transport in two-terminal waveguides with a spin-orbital interaction and magnetic field modulations. Phys Rev Lett 94:246601.

[89] Silvestrov PG, Mishchenko EG (2006) Polarized electric current in semiclassical transport with spin-orbit interaction. Phys Rev B 74:165301.

[90] van Wees BJ, van Houten H, Beenakker CWJ, Williamson JG, Kouwenhoven LP, van der Marel D, Foxon CT (1988) Quantized conductance of point contacts in a two-dimensional electron gas. Phys Rev Lett 60:848-850.

[91] Wharam DA, Thornton TJ, Newbury R, Pepper M, Ahmed H, Frost JEF, Hasko DG, Peacock DC, Ritchie DA, Jones GAC (1988) One-dimensional transport and the quantisation of the ballistic resistance. J Phys C: Solid State Phys 21:L209-L214.

[92] Sánchez D, Serra L (2006) Fano-Rashba effect in a quantum wire. Phys Rev B 74:153313.

[93] Dresselhaus G (1955) Spin-orbit coupling effects in zinc blende structures. Phys Rev 100:580-586.

[94] Bardarson JH, Adagideli I, Jacquod P (2007) Mesoscopic spin Hall effect. Phys Rev Lett 98:196601.

[95] Hankiewicz EM, Molenkamp LW, Jungwirth T, Sinova J (2004) Manifestation of the spin Hall effect through chargetransport in the mesoscopic regime. Phys Rev B 70:241301.

[96] Sheng L, Sheng DN, Ting CS (2005) Spin-Hall effect in two-dimensional electron systems with Rashba spin-orbit coupling and disorder. Phys Rev Lett 94:016602.

[97] Governale M, Taddei F, Fazio R (2003) Pumping spin with electrical fields. Phys Rev B 68:155324.

[98] Sharma P, Brouwer PW (2003) Mesoscopic effects in adiabatic spin pumping. Phys Rev Lett 91:166801.

[99] Mucciolo ER, Chamon C, Marcus CM (2002) Adiabatic quantum pump of spin-polarized current. Phys Rev Lett 89:146802.

[100] Watson SK, Potok RM, Marcus CM, Umansky V (2003) Experimental realization of a quantum spin pump. Phys Rev Lett 91:258301.

[101] Reimann P (2002) Brownian motors: noisy transport far from equilibrium. Phys Rep 361:57-265.

[102] Scheid M, Wimmer M, Bercioux D, Richter K (2006) Zeeman ratchets for ballistic spin currents. Phys Status Solidi (c) 3:4235.

[103] McLennan MJ, Lee Y, Datta S (1991) Voltage drop in mesoscopic systems: A numerical study using a quantum kinetic equation. Phys Rev B 43:13846-13884.

[104] Ohno H, Shen A, Matsukura F, Oiwa A, Endo A, Katsumoto S, Iye Y (1996) (Ga,Mn)As: A new diluted magnetic semiconductor based on GaAs. Appl Phys Lett 69:363-365.

[105] König M, Wiedmann S, Brüne C, Roth A, Buhmann H, Molenkamp LW, Qi XL, Zhang SC (2007) Quantum spin Hall insulator state in HgTe quantum wells. Science 318:766-770.

[106] Cuniberti G, Fagas G, Richter K (eds) (2006) Introducing Molecular Electronics. Springer, Berlin, Heidelberg.

[107] Emberly E, Kirczenow G (2002) Molecular spintronics: spin-dependent electron transport in molecular wires. Chemical Physics 281:311-324.

[108] Novoselov KS, Geim AK, Morozov SV, Jiang D, Zhang Y, Dubonos SV, Grigorieva IV, Firsov AA (2004) Electric field effect in atomically thin carbon films. Science 306:666-669.

[109] Russo S, Oostinga JB, Wehenkel D, Heersche HB, Sobhani SS, Vandersypen LMK, Morpurgo AF (2007) Aharonov-Bohm effect in graphene. arXiv:0711.1508v1.

[110] Huertas-Hernando D, Guinea F, Brataas A (2006) Spin-orbit coupling in curved graphene, fullerenes, nanotubes, and nanotube caps. Phys Rev B 74:155426.

[111] Min H, Hill JE, Sinitsyn NA, Sahu BR, Kleinman L, MacDonald AH (2006) Intrinsic and Rashba spin-orbit interactions in graphene sheets. Phys Rev B 74:165310.

[112] Wimmer M, Adagideli I, Berber S, Tománek D, Richter K (2007) Spin transport in rough graphene nanoribbons. 
arXiv:0709.3244v1.

Books and reviews

Datta S (2002) Electronic Transport in Mesoscopic Systems. Cambridge University Press, Cambridge.

Ferry DK, Goodnick SM (2001) Transport in Nanostructures. Cambridge University Press, Cambridge.

Bruus H, Flensberg K (2004) Many-body Quantum Theory in Condensed Matter Physics: An Introduction. Oxford University Press, Oxford.

Fabian J, Matos-Abiague A, Ertler C, Stano P, Žutić I (2007) Semiconductor Spintronics. Acta Physica Slovaca 57:565-907. 